This item was submitted to Loughborough's Research Repository by the author.

Items in Figshare are protected by copyright, with all rights reserved, unless otherwise indicated.

\title{
On mechanisms of destruction of adiabatic invariance in slow-fast Hamiltonian systems
}

PLEASE CITE THE PUBLISHED VERSION

https://doi.org/10.1088/1361-6544/ab2a2c

\section{PUBLISHER}

(C) IOP Publishing Ltd and London Mathematical Society

\section{VERSION}

AM (Accepted Manuscript)

\section{PUBLISHER STATEMENT}

Reproduced with permission. All rights reserved.

LICENCE

All Rights Reserved

\section{REPOSITORY RECORD}

Neishtadt, Anatoly. 2019. "On Mechanisms of Destruction of Adiabatic Invariance in Slow-fast Hamiltonian Systems”. figshare. https://hdl.handle.net/2134/38092. 


\title{
On mechanisms of destruction of adiabatic invariance in slow-fast Hamiltonian systems
}

\author{
Anatoly Neishtadt ${ }^{1,2, *}$ \\ ${ }^{1}$ Loughborough University, Loughborough, LE11 3TU, UK \\ ${ }^{2}$ Space Research Institute, Moscow, 117997, Russia
}

\begin{abstract}
In many problems of classical mechanics and theoretical physics dynamics can be described as a slow evolution of periodic or quasi-periodic processes. Adiabatic invariants are approximate first integrals for such a dynamics. Existence of adiabatic invariants makes dynamics close to regular. Destruction of adiabatic invariance leads to chaotic dynamics. We present a review of some mechanisms of destruction of adiabatic invariance in slow-fast Hamiltonian systems with examples from charged particle dynamics.
\end{abstract}

\section{Preliminaries: conservation of adiabatic invariance}

\subsection{One-frequency systems with slowly varying parameters}

Adiabatic invariant is an approximate first integral (conservation law) of a system with slow and fast variables (slow-fast system). A classical example of an adiabatic invariant is the "action" variable of a linear oscillator with slowly varying frequency. Equation of motion of such an oscillator is

$$
\ddot{q}+\omega^{2}(\tau) q=0, \dot{\tau}=\varepsilon, 0<\varepsilon \ll 1 .
$$

The energy of the oscillator is

$$
E=E(\dot{q}, q, \tau)=\frac{1}{2}\left(\dot{q}^{2}+\omega^{2}(\tau) q^{2}\right) .
$$

For frozen value of $\omega$ one can consider the phase portrait of this oscillator (Fig. 1) in the plane $q, p=\dot{q}$.

${ }^{*}$ E-mail: A.Neishtadt@lboro.ac.uk 


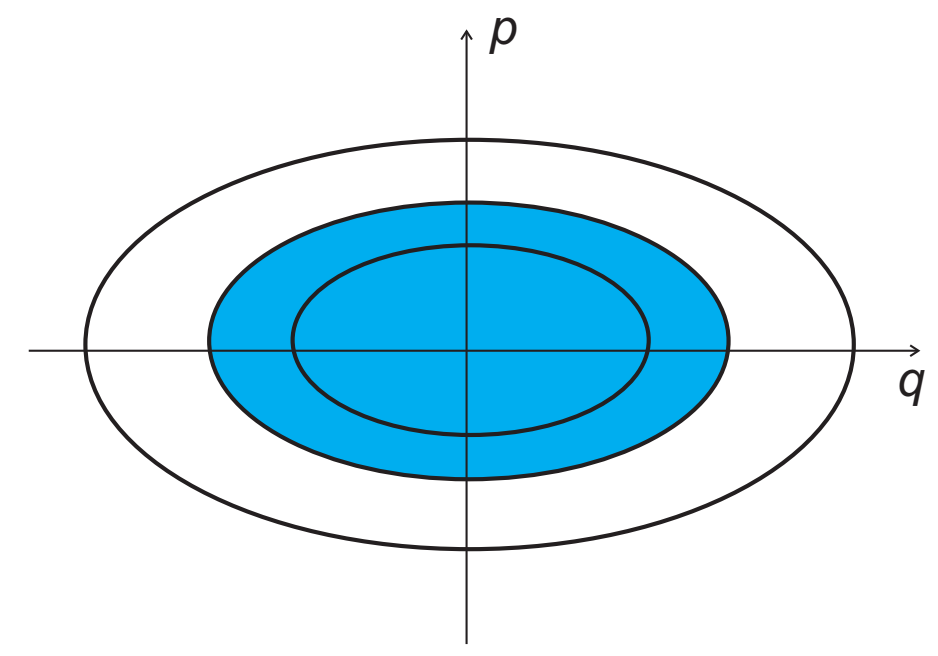

Figure 1. Phase portrait of a linear oscillator.

The "action" variable in this portrait (the area surrounded by a trajectory, divided by $2 \pi$ ) is $I(p, q, \tau)=E(p, q, \tau) / \omega(\tau)$. For equation (1.1) the value of action along a trajectory remains approximately constant on the time interval $0 \leq t \leq 1 / \varepsilon$ :

$$
I(p(t), q(t), \varepsilon t)=I(p(0), q(0), 0)+O(\varepsilon),
$$

where $(p(t), q(t))$ is a solution of the equation (1.1), see, e.g., [4], Sect. 52.F). Under rather general assumptions this estimate is valid on much longer time intervals ([5], Sect. 6.4.4).

Adiabatic invariants are usually first integrals of some averaged system. For example, consider a Hamiltonian system with one degree of freedom and a dependence on a parameter. The Hamiltonian is $E=E(p, q, \tau)$, where $p, q$ are conjugate phase variables, and $\tau$ is a parameter. Assume that in the phase portrait of this system there is a domain filled by closed trajectories, similarly to Fig. 1. In this domain one can introduce the action-angle variables $I, \varphi \bmod 2 \pi$ by a canonical transformation $p, q \mapsto I, \varphi$. Make the same canonical transformation when $\tau$ changes slowly in time, $\dot{\tau}=\varepsilon$. Then the dynamics of the variables $I, \varphi$ is described by the Hamiltonian system with the Hamiltonian $H=H_{0}(I, \tau)+\varepsilon H_{1}(I, \varphi, \tau)$, where $H_{0}$ is the function $E$ expressed via $I, \tau$, and $H_{1}$ is $2 \pi$-periodic in $\varphi$ (see, e.g., [4], Sect. 52.F):

$$
\dot{I}=-\varepsilon \frac{\partial H_{1}}{\partial \varphi}, \quad \dot{\varphi}=\frac{\partial H_{0}}{\partial I}+\varepsilon \frac{\partial H_{1}}{\partial I}, \quad \dot{\tau}=\varepsilon .
$$

This system is in a standard form for application of the averaging method [11] with the fast rotating phase $\varphi$ and the slow variables $I, \tau$. The averaged over $\varphi$ equation for $I$ is $\dot{I}=0$, i.e. $I$ is a first integral of the averaged equations. Standard theorems about accuracy of the averaging method imply approximate conservation of $I$ on long enough time intervals (see details in [5], Sect. 6.4.4). Thus $I$ is an adiabatic invariant. 


\subsection{Slow-fast Hamiltonian systems}

Consider a Hamiltonian system with the Hamiltonian $E=E(p, q, y, x)$ and the symplectic structure $\Omega=d p \wedge d q+\varepsilon^{-1} d y \wedge d x$. Here $(p, q) \in \mathbb{R}^{2 n},(y, x) \in \mathbb{R}^{2 m}$. The equations of motion are

$$
\dot{p}=-\frac{\partial E}{\partial q}, \dot{q}=\frac{\partial E}{\partial p}, \dot{y}=-\varepsilon \frac{\partial E}{\partial x}, \dot{x}=\varepsilon \frac{\partial E}{\partial y} .
$$

Here $p, q$ are called fast variables, $y, x$ are called slow variables. Study of systems with slowly varying in time parameter can be reduced to this case by taking this parameter as a new coordinate and introducing the conjugate momentum.

Consider the particular case when $n=1$, i. e. we have one degree of freedom for fast variables. As the variables $y, x$ change slowly, it is reasonable to consider the problem at frozen values of $y, x$ first. The system for the fast variables with frozen slow variables is called a fast system. Assume that for all frozen values of $y, x$ there is a domain filled by closed phase curves of Hamiltonian $E$ in the plane $p, q$, like in Fig. 1. In this domain one can introduce the "action-angle" variables $I=I(p, q, y, x), \varphi=\varphi(p, q, y, x) \bmod 2 \pi$. Denote $H_{0}(I, y, x)$ the Hamiltonian $E$ expressed via the variables $I, y, x$. Then one can write the equations of motion in the exact system for variables $I, \varphi, y, x$ and perform averaging over $\varphi$. This leads to the following adiabatic approximation:

$$
I=\mathrm{const}, \quad \dot{y}=-\varepsilon \frac{\partial H_{0}}{\partial x}, \quad \dot{x}=\varepsilon \frac{\partial H_{0}}{\partial y} .
$$

These relations describe the actual dynamics with an accuracy $O(\varepsilon)$ on time intervals of length of order $1 / \varepsilon$. Typically, the relation $I=$ const is valid with an accuracy $O(\varepsilon)$ on much longer time intervals ([5], Sect. 6.4.4).

\subsection{Improved adiabatic invariant}

Typical behavior of the action variable $I$ in system (1.2) is shown in Fig. 2, (a). The variable $I$ oscillates with an amplitude of order $\varepsilon$ about a constant value. A procedure called adiabatic perturbation theory allows to introduce an improved adiabatic invariant $J=I+\varepsilon u(I, \varphi, y, x)$ such that $J$ oscillates with an amplitude of order $\varepsilon^{2}$ about a constant value (Fig. 2, (b)). Here $u$ is $2 \pi$-periodic in $\varphi$. One can also introduce an improved adiabatic invariant of $n$th order, $n \geq 1$, whose value oscillates with an amplitude $O\left(\varepsilon^{n}\right)$.

\subsection{Perpetual conservation of an adiabatic invariant}

Consider system (1.2) for 2 degrees of freedom: $m=n=1$, i. e. we have one degree of freedom for fast variables, and one degree of freedom for slow variables. Consider motion in 

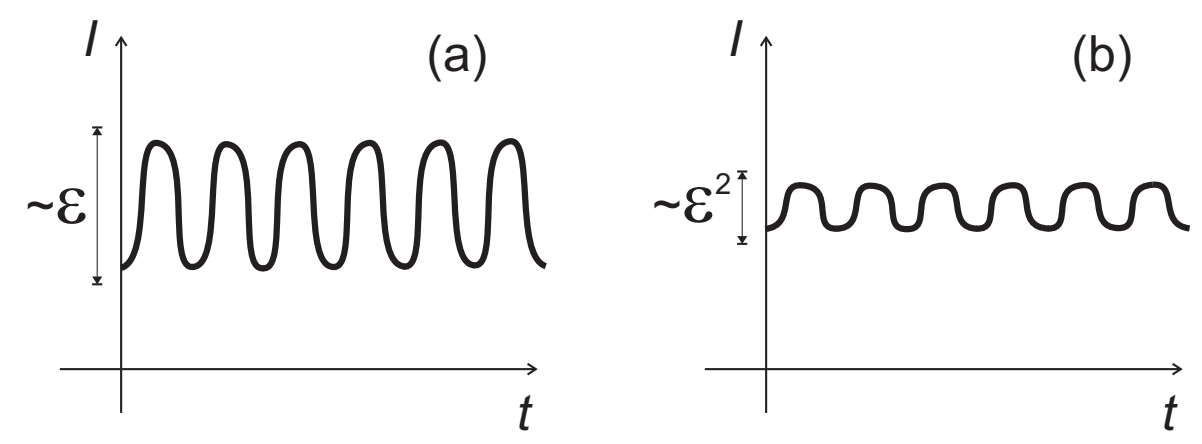

Figure 2. Usual and improved adiabatic invariants.

an energy level $E=h=$ const. As in Subsection 1.2, we assume that for all frozen values of slow variables $y, x$ there is a domain filled by closed phase curves of the Hamiltonian $E$ in the plane of fast variables $p, q$. In the adiabatic approximation (1.3) the dynamics of slow variables is described by the Hamiltonian system with one degree of freedom and the Hamiltonian $H_{0}(I, y, x), I=$ const. Assume that there is a domain filled by closed phase curves of this Hamiltonian in the phase plane of slow variables. In the adiabatic approximation, the motion looks as follows. In the plane of slow variables, the phase point moves in a closed curve, and, in the plane of fast variables, the phase point moves in a slowly periodically pulsing curve determined by the condition $I=$ const. Thus, in the 3 dimensional energy level $E=h$ there is a domain filled by 2-dimensional invariant tori of motion in adiabatic approximation. It was shown by V.I. Arnold [2], that under some nondegeneracy condition the energy level $E=h$ is filled by invariant tori of the exact system up to a residue of a small measure. These tori are $O(\varepsilon)$-close to the invariant tori of the adiabatic approximation. Therefore the phase point of the exact system either moves in a torus $O(\varepsilon)$-close to some torus $I=$ const, or moves in an $O(\varepsilon)$-narrow gap between tori with $I=$ const $+O(\varepsilon)$. (Actually, in analytic systems this gap is even exponentially narrow [5], Sect. 6.3.3.) A corollary of this is the V.I. Arnold theorem about perpetual adiabatic invariance: for all initial conditions, the variation of $I$ along the trajectory of the phase point is small on the infinite time interval [2]:

$$
|I(t)-I(0)|=O(\varepsilon) \text { for }-\infty<t<+\infty .
$$

This statement has many applications in the theory of motion of charged particles. In particular, it implies that it is possible to perpetually confine charged particles in axisymmetric magnetic adiabatic traps [2]. 


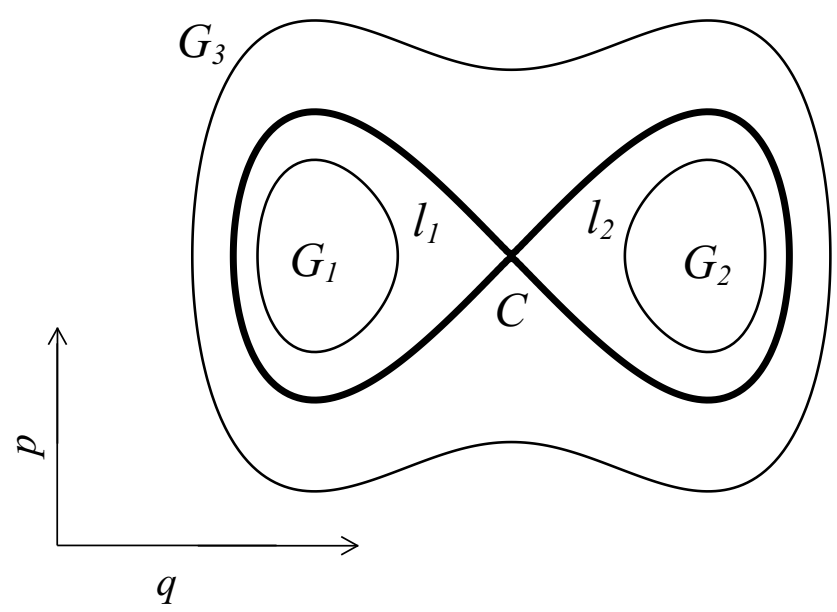

Figure 3. Phase portrait of the fast system.

\section{Passage through a separatrix}

\subsection{Description of a separatrix crossing}

Consider, as in the previous Section, a slow-fast Hamiltonian system with two degrees of freedom. However, assume now that the phase portrait of the fast system has the form shown in Fig. 3 for $(x, y) \in \Sigma$, where $\Sigma$ is some domain in $\mathbb{R}^{2}$. The unstable stationary point $C$ is a non-degenerate saddle point. Denote $h_{c}=h_{c}(y, x)$ the value of the Hamiltonian $E$ at the point $C$. The separatrices $l_{1}=l_{1}(y, x)$ and $l_{2}=l_{2}(y, x)$ divide the phase portrait into three regions $G_{\nu}=G_{\nu}(y, x), \nu=1,2,3$. Consider the motion in an energy level $E=h$. Denote $\Sigma_{i}, i=1,2,3$ the set of points $(x, y) \in \Sigma$ such that in the domain $G_{i}$ there is an energy level curve on which $E(p, q, y, x)=h$. The set $\Sigma_{i}$ is the phase space of the system of adiabatic approximation for the domain $G_{i}$. A part of the boundary of $\Sigma_{i}$ is the curve $\Gamma_{i}$ which is formed by the points $(x, y)$ such that $h_{c}(y, x)=h$. Let us identify (glue together) $\Gamma_{1}, \Gamma_{2}$ and $\Gamma_{3}$. Denote $\Gamma$ the obtained curve (it is called an uncertainty curve [41]). Thus $\Sigma_{1}, \Sigma_{2}$ and $\Sigma_{3}$ are glued along $\Gamma$. The obtained singular manifold $\tilde{\Sigma}$ is the phase space of the system in the adiabatic approximation. Thus $\tilde{\Sigma}=\Sigma_{1} \cup \Sigma_{2} \cup \Sigma_{3} \cup \Gamma$, Fig. 4. Dynamics in the adiabatic approximation in $\Sigma_{i}$ is described by equations (1.3), where the function $H_{0}$ is calculated for the domain $G_{i}$. In this domain the value $I$ is a function of the value of energy $h$ and slow variables: $I=\mathcal{I}(h, y, x)$. On the trajectory of system (1.3) in the domain $\Sigma_{i}$ we have $\mathcal{I}(h, y, x)=$ const.

In order to describe separatrix crossing in the adiabatic approximation we need some additional notation. Denote $\mathcal{I}_{i}$ the function $\mathcal{I}$ for the domain $\Sigma_{i}$. Denote $S_{i}(y, x)$ the area of the domain $G_{i}, i=1,2$. Let $S_{3}(y, x)=S_{1}(y, x)+S_{2}(y, x)$. Denote $\Theta_{i}(y, x)=\left\{S_{i}(y, x), h_{c}(y, x)\right\}$, 


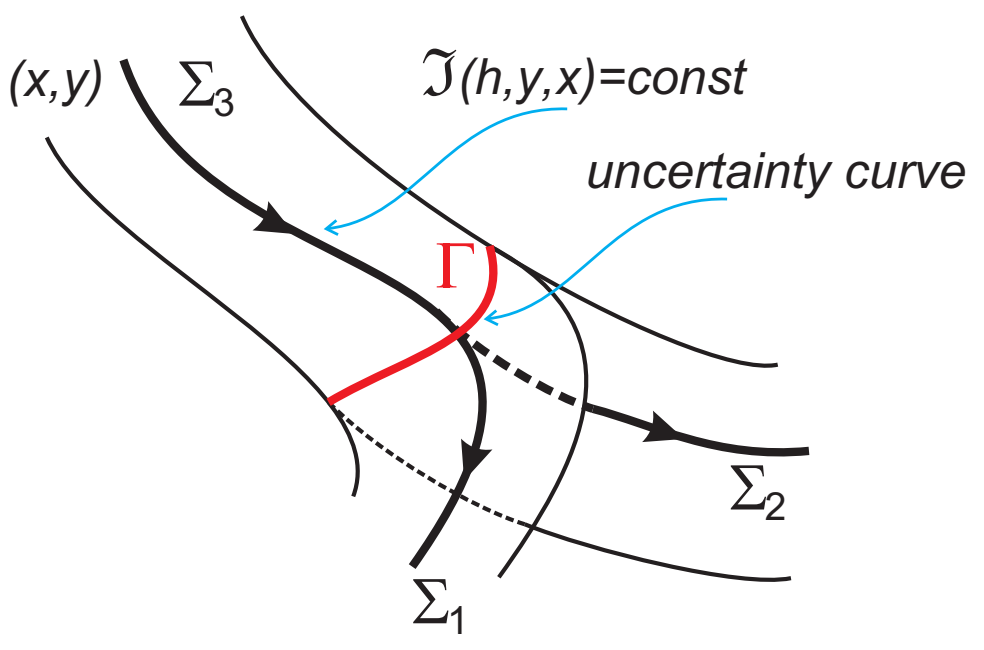

Figure 4. A piece of the phase space of slow system in the energy level $E(p, q, y, x)=h$.

where $\{\cdot, \cdot\}$ is the Poisson bracket in the variables $y, x:\{f, g\}=\frac{\partial f}{\partial x} \frac{\partial g}{\partial y}-\frac{\partial f}{\partial y} \frac{\partial g}{\partial x}$. Let a trajectory of the adiabatic approximation in the domain $\Sigma_{3}$ arrive at the curve $\Gamma$ at a point $\left(x_{*}, y_{*}\right)$. Assume that $\Theta_{i}\left(y_{*}, x_{*}\right)>0, i=1,2,3$. The trajectory has two continuations: in the domain $\Sigma_{1}$ and in the domain $\Sigma_{2}$. Probability $P_{i}(y, x)=\frac{\Theta_{i}\left(y_{*}, x_{*}\right)}{\Theta_{3}\left(y_{*}, x_{*}\right)}$ is assigned to continuation in the domain $\Sigma_{i}{ }^{1}$

Passage through a separatrix leads to a jump in an adiabatic invariant. This is seen already in the adiabatic approximation. Let $I_{3,0}$ be the value of $I$ on a trajectory of the adiabatic approximation in the domain $\Sigma_{3}$. Let this trajectory arrive at the curve $\Gamma$ at a point $\left(x_{*}, y_{*}\right)$. Then $2 \pi S_{3}\left(y_{*}, x_{*}\right)=I_{3,0}$. For the continuation of this trajectory in the domain $\Sigma_{i}, i=1,2$ we have $\mathcal{I}_{i}=2 \pi S_{i}\left(y_{*}, x_{*}\right)$. This jump in the value of $I$ occurs due to the change of geometry of the unperturbed trajectory. We call it a geometrical jump of the adiabatic invariant.

Consider now a trajectory of the exact system (1.2) on a time interval $0 \leq t \leq K / \varepsilon$, $K=$ const. Let the initial point of this trajectory correspond to the domain $G_{3}$, and the final point correspond to one of the domains $G_{i}, i=1,2$. Denote $I_{3,0}, J_{3,0}$ and $I_{i, K}, J_{i, K}$ the initial and the final values of the adiabatic invariant $I$ and of the improved adiabatic invariant $J$. Determine $\left(x_{*}, y_{*}\right)$ as in the previous paragraph. As an adiabatic prediction of the value $J_{i, K}$ we consider

$$
J_{i, K, a}=\frac{1}{2 \pi} S_{i}\left(x_{*}, y_{*}\right)+\frac{\Theta_{i}\left(y_{*}, x_{*}\right)}{\Theta_{3}\left(y_{*}, x_{*}\right)}\left(J_{3,0}-\frac{1}{2 \pi} S_{3}\left(x_{*}, y_{*}\right)\right) .
$$

We call the value $\Delta J_{i}=J_{i, K}-J_{i, K, a}$ a dynamical jump of the adiabatic invariant. There are

\footnotetext{
${ }^{1}$ Such a probabilistic approach was introduced in [25] for a problem of motion of quasiparticles. It was reinvented in several other contexts independently afterwards, see, in particular [22, 23]. Several approaches to rigorous definition of probability in the considered deterministic problem are possible: see [2, 20, 42] and a discussion in [30]. We use an approach in [2]: probability of some event is determined by the measure of the initial conditions for this event. For a detailed proof in the case of slow-fast systems see [30].
} 
asymptotic formulas for this value ([39] for the pendulum in a slowly varying gravitational field, then $[14,26]$ for the general case of a Hamiltonian system with one degree of freedom and slowly varying parameters, [27] for the general case of a slow-fast Hamiltonian system with two degrees of freedom). The leading terms of order $\varepsilon \ln \varepsilon$ and $\varepsilon$ are calculated. The term of order $\varepsilon \ln \varepsilon$ vanishes in a symmetric situation when $\Theta_{1}\left(y_{*}, x_{*}\right)=\Theta_{2}\left(y_{*}, x_{*}\right)$.

Problems with a separatrix crossing require a probabilistic approach because of the sensitive dependence on initial conditions of the outcome of separatrix crossing for small $\varepsilon$. A change of order $\varepsilon$ in initial conditions may change this outcome: the phase point will continue its motion in $G_{2}$ instead of $G_{1}$ or vice versa. Thus, the question about the outcome of separatrix crossing does not have a deterministic answer in the limit as $\varepsilon \rightarrow 0$. However, it is possible to consider the probabilities of different outcomes and to calculate these probabilities. Similarly, a dynamical jump of the adiabatic invariant has a sensitive dependence on initial conditions. A change of order $\varepsilon$ in initial conditions produces a relative change of order 1 in the value of the dynamical jump. It is possible to consider the probabilistic distribution of the dynamical jump of adiabatic invariant. Formulas in $[27,35]$ allow to calculate this distribution.

Accumulation of results of geometrical and dynamical jumps of the adiabatic invariant at multiple separatrix crossings leads to a destruction of adiabatic invariance. Below we provide examples of such a destruction.

\subsection{Destruction of adiabatic invariance due to dynamical jumps of the adiabatic invariant in systems with a symmetry}

Consider, following [12, 43], the motion of a charged particle in the parabolic model of a planetary magnetotail. The dynamics of such a particle is described by the slow-fast Hamiltonian system with the Hamiltonian

$$
E=\frac{1}{2} y^{2}+\frac{1}{2} p^{2}+\frac{1}{2}\left(x-\frac{1}{2} q^{2}\right)^{2}
$$

with fast variables $p, q$, slow variables $y, x$, and small parameter $\varepsilon$. The meaning of the variables is explained in [12]. The phase portrait of the fast system is shown in Fig. 5, (a) for $x<0$ and in Fig. 5, (b) for $x>0$.

Separatrices in Fig.5, (b) divide the phase portrait into domains $G_{1}, G_{2}, G_{3}$. The phase portrait is symmetric with respect to the coordinate axis $q=0$. (It is symmetric also with respect to the axis $p=0$, but this is not essential here.) The phase portrait of the slow system in the energy level $\left\{E=\frac{1}{2}\right\}$ is shown in Fig. 6 (the phase portrait in any other energy level can be obtained by a rescaling).

Let $\Sigma_{1,2.3}$ be as in the previous Section, $\Sigma_{0}$ be the domain of slow variables where the phase portrait of the fast motion has the form shown in Fig. 5, (a). The slow dynamics takes place 

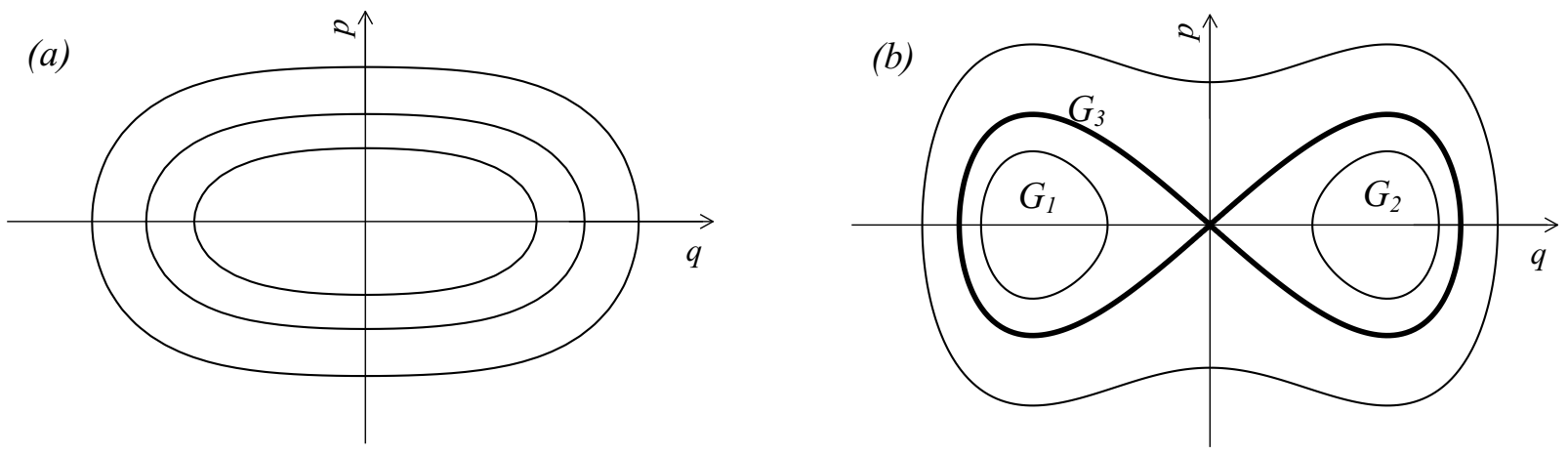

Figure 5. Phase portraits of the fast system for motion of a charged particle in the parabolic model of a planetary magnetotail.

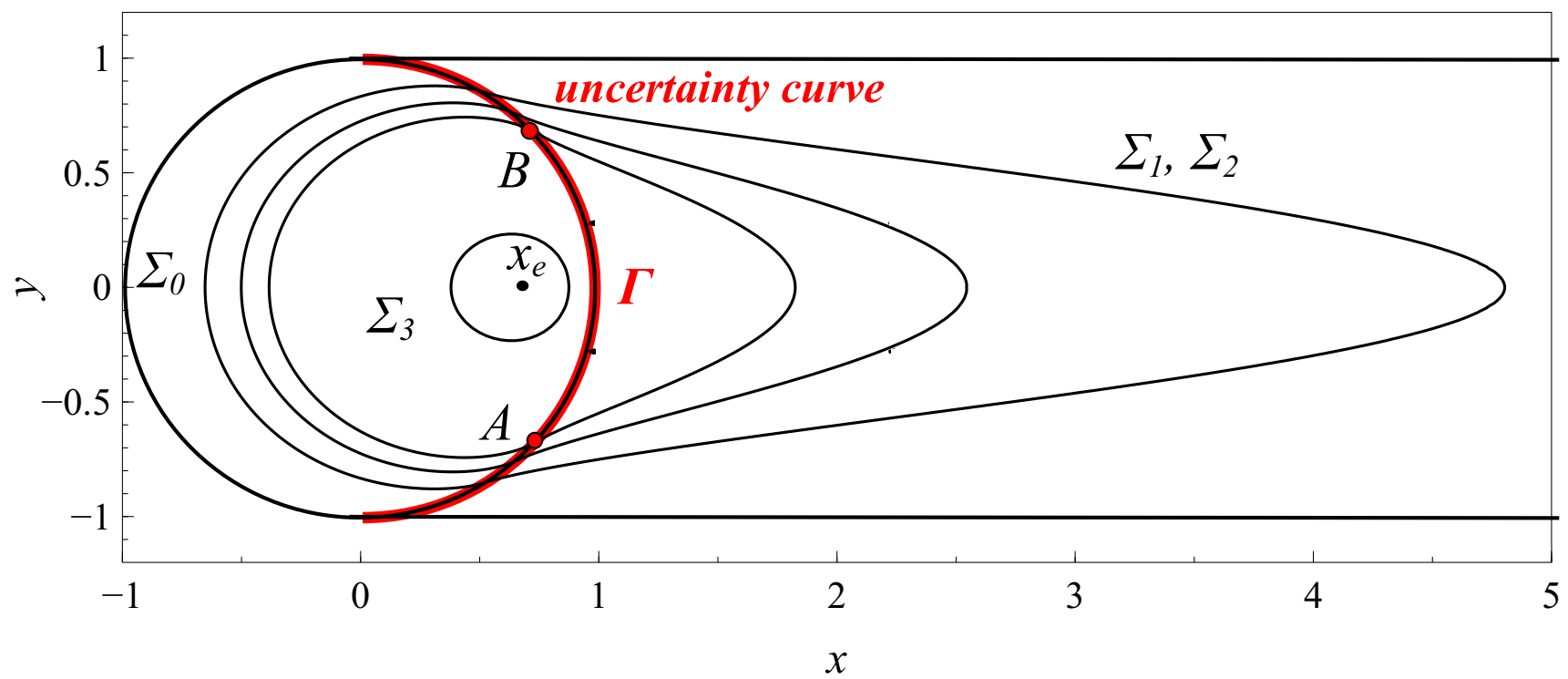

Figure 6. The phase portrait of the slow system in an energy level for motion of a charged particle in the parabolic model of a planetary magnetotail. 


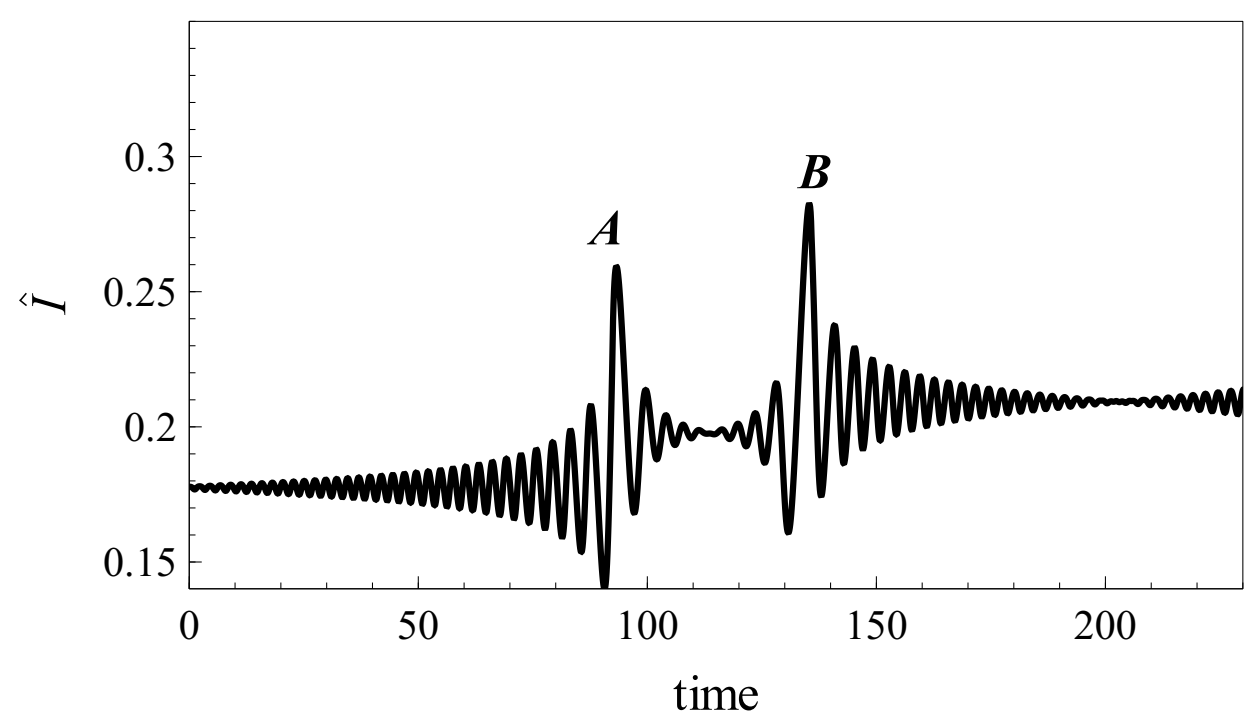

Figure 7. Two jumps of an adiabatic invariant.

in the domain bounded by the rays $\{y= \pm 1, x \geq 0\}$ and the semicircle $\left\{x^{2}+y^{2}=1, x \leq 0\right\}$. The semicircle $\Gamma=\left\{x^{2}+y^{2}=1, x \geq 0\right\}$ is the uncertainty curve. In the notation of the previous Section we have $\Sigma_{3}=\left\{x^{2}+y^{2}<1, x>0\right\}$. The domains $\Sigma_{1}$ and $\Sigma_{2}$ are shown as one domain $\left\{|y| \leq 1, x^{2}+y^{2}>1\right\}$. In Fig. 6 the trajectories of the slow system look like closed curves. However, in the phase space of the adiabatic approximation $\tilde{\Sigma}=\Sigma_{0} \cup \Sigma_{1} \cup \Sigma_{2} \cup \Sigma_{3} \cup \Gamma$ there is a branching on the curve $\Gamma$ (cf. Fig. 13 in [12]). The continuations to $\Sigma_{1}$ and $\Sigma_{2}$ at this branching have equal probabilities $1 / 2$. Introduce the function $\hat{I}$ which coincides with $I$ in the domains $G_{1,2}$ and equals $I / 2$ in the domain $G_{3}$. The function $\hat{I}$ is continuous at the separatrices. Thus, there is no geometrical jump in $\hat{I}$. Along the adiabatic trajectory $\hat{I}=$ const. Along the exact trajectory $\hat{I}$ oscillates. Passage through a separatrix leads to a dynamical jump in $\hat{I}$. Fig. 7 shows $\hat{I}$ as a function of time $t$ for a fragment of the exact trajectory with two separatrix crossings. Fig. 8 shows the projection of this fragment of the trajectory onto the plane of slow variables. Passage through a separatrix leads to a dynamical jump in $\hat{I}$ (in Fig. 7 the points A and B correspond to separatrix crossings). Fig. 9 shows the projection of a long trajectory onto the plane of slow variables.

It is seen that the accumulation of results of dynamical jumps of the adiabatic invariant leads to a diffusion across level lines of the adiabatic invariant and destruction of adiabatic invariance. Each jump of the adiabatic invariant can be considered as a random value with the mean 0 and the standard deviation of order $\varepsilon$. Time between subsequent separatrix crossings is of order $1 / \varepsilon$. Thus, under the hypothesis of the statistical independence of the sequence of jumps of adiabatic invariant we could estimate that diffusion at a distance $\sim 1$ takes time $\sim 1 / \varepsilon^{3}$. However, subsequent jumps of the adiabatic invariant can not be considered as statistically independent in problems with a symmetry similar to considered here $[15,16]$. Moreover, consider the domain of the phase space where separatrix crossings 


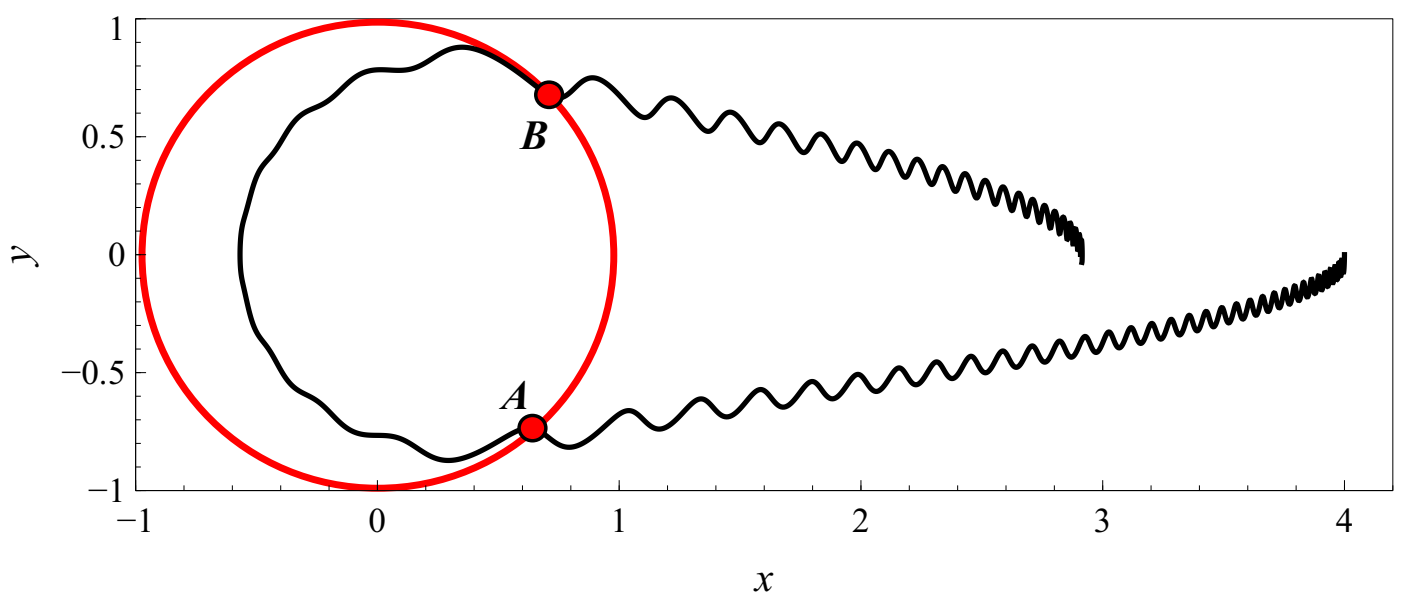

Figure 8. Projection of a trajectory with two separatrix crossings onto the plane of slow variables.

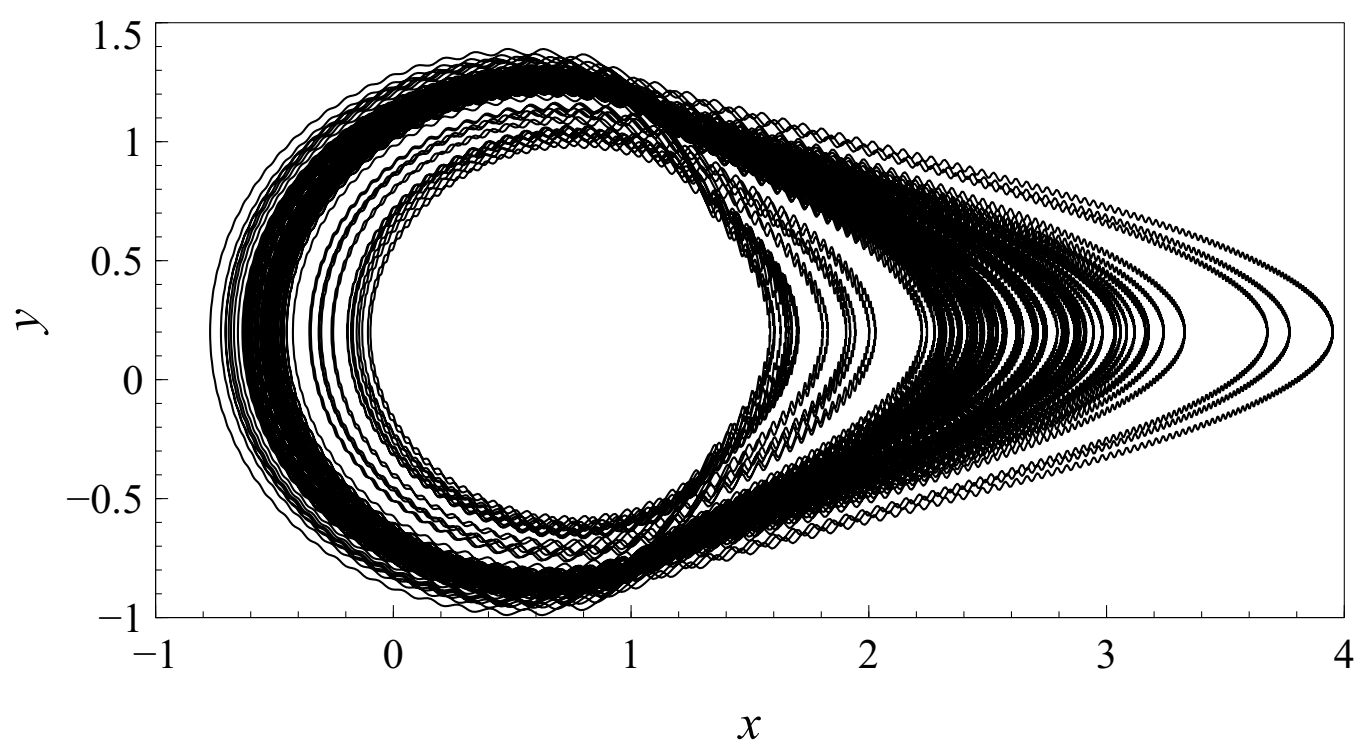

Figure 9. Long term dynamics in the plane of slow variables. 

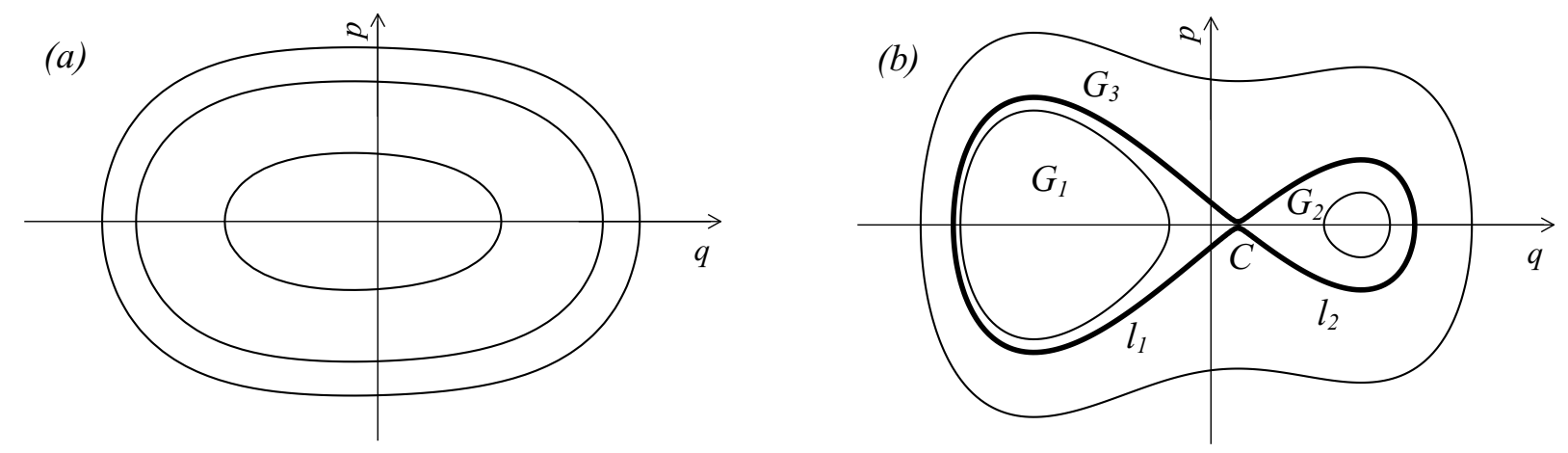

Figure 10. Phase portraits of fast system for motion of a charged particle in a model of a non-symmetric planetary magnetotail.

are possible. Visually, this domain looks as a "chaotic sea" for small $\varepsilon$. However, this domain contains many, $\sim 1 / \varepsilon$, stability islands, and measure of each of these islands is bounded below by a value $\sim \varepsilon[32,33]$. Thus the total measure of the stability islands remains bounded below by a value that does not depend on $\varepsilon$. The estimate $\sim 1 / \varepsilon^{3}$ for the diffusion time is probably correct for a large measure of initial data because the total measure of the stability islands is small even if it does not tend to 0 as $\varepsilon \rightarrow 0$.

\subsection{Destruction of adiabatic invariance due to geometrical jumps of adiabatic invariant}

Geometrical jumps of adiabatic invariant provide a mechanism of rather fast destruction of adiabatic invariance. As an example, consider, following [9], the motion of a charged particle in a model of planetary magnetotail taking into account an asymmetry of the magnetic field with respect to the equatorial plane of the planet and a presence of an electric field. Dynamics of the particle is described by the slow-fast Hamiltonian system with the Hamiltonian

$$
E=\frac{1}{2}(y-s q)^{2}+\frac{1}{2} p^{2}+\frac{1}{2}\left(x-\frac{1}{2} q^{2}\right)^{2}-s q u
$$

with fast variables $p, q$, slow variables $y, x$, positive constants $s, u$, and small parameter $\varepsilon$. The meaning of the variables is explained in [9]. Two possible types of phase portraits of the fast system are shown in Fig. 10, (a),(b). Separatrices in Fig. 10, (b) divide the phase portrait into domains $G_{1}, G_{2}, G_{3}$. Let the corresponding domains in the phase space of the slow system in the energy level $\left\{E=\frac{1}{2}\right\}$ be $\Sigma_{1,2,3}$ determined as in Section 2.2. Let $\Sigma_{0}$ be the domain in this phase space where the phase portrait of the fast motion has the form shown in Fig. 10, (a). 


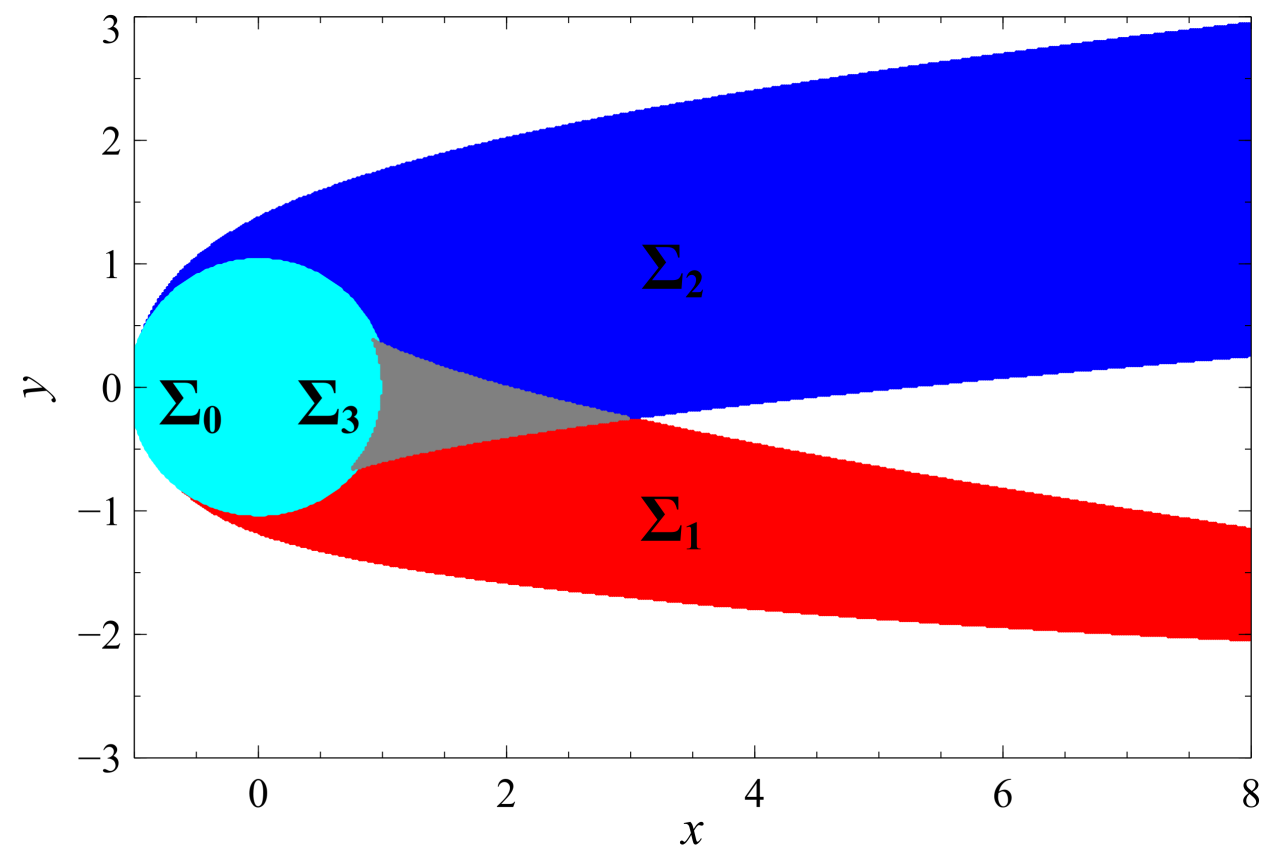

Figure 11. (Color online.) Projection of the phase space of the slow system onto the plane of slow variables for motion of a charged particle in a model of a non-symmetric planetary magnetotail.

Fig. 11 shows the projection of the phase space of the slow system onto the plane of slow variables. Values $x, y$ in the grey domain have pre-images both in $\Sigma_{1}$ and $\Sigma_{2}$. Thus, the phase portrait of the slow system can not be shown in this plane (two different directions of the vector field of the slow system correspond to each point in the grey domain). Fig. 12 shows $I$ as a function of time $t$ for a fragment of the exact trajectory with two separatrix crossings.

Each separatrix crossing leads to a big $\sim 1$ jump in the adiabatic invariant. Fig. 13 shows the projection of a long trajectory onto the plane of slow variables. On can see a complicated dynamics created by separatrix crossings.

There are also intermediate cases between those for Hamiltonians (2.1) and (2.2). For these cases, solutions of a slow system travel between a certain number of values of the adiabatic invariant due to a certain symmetry in the slow system. Dynamical jumps of the adiabatic invariant produce a slow diffusion around these values. Such process occurs, e.g., for Hamiltonian (2.2) with $u=0$ [8]. This mechanism of destruction of adiabatic invariance plays a basic role in multi-turn extraction of charged particles in accelerators [13]. 


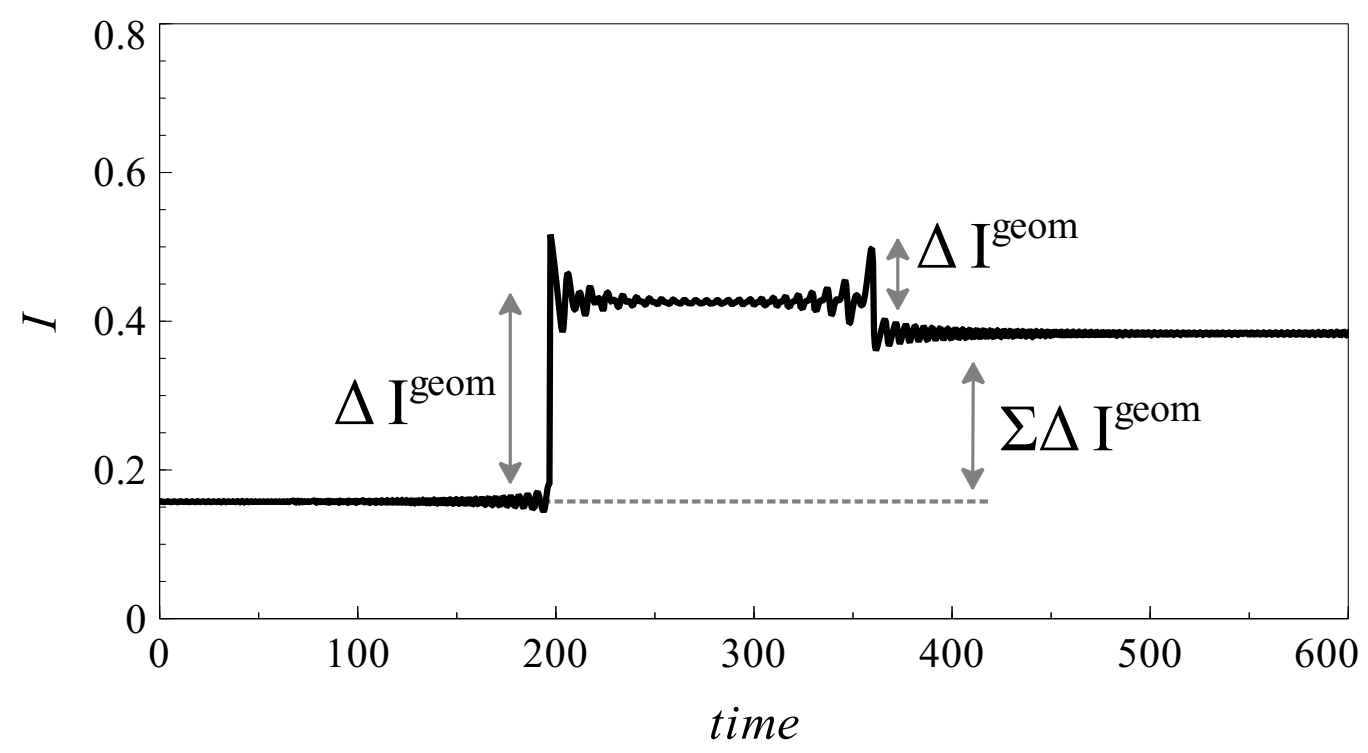

Figure 12. Two geometrical jumps of the adiabatic invariant.

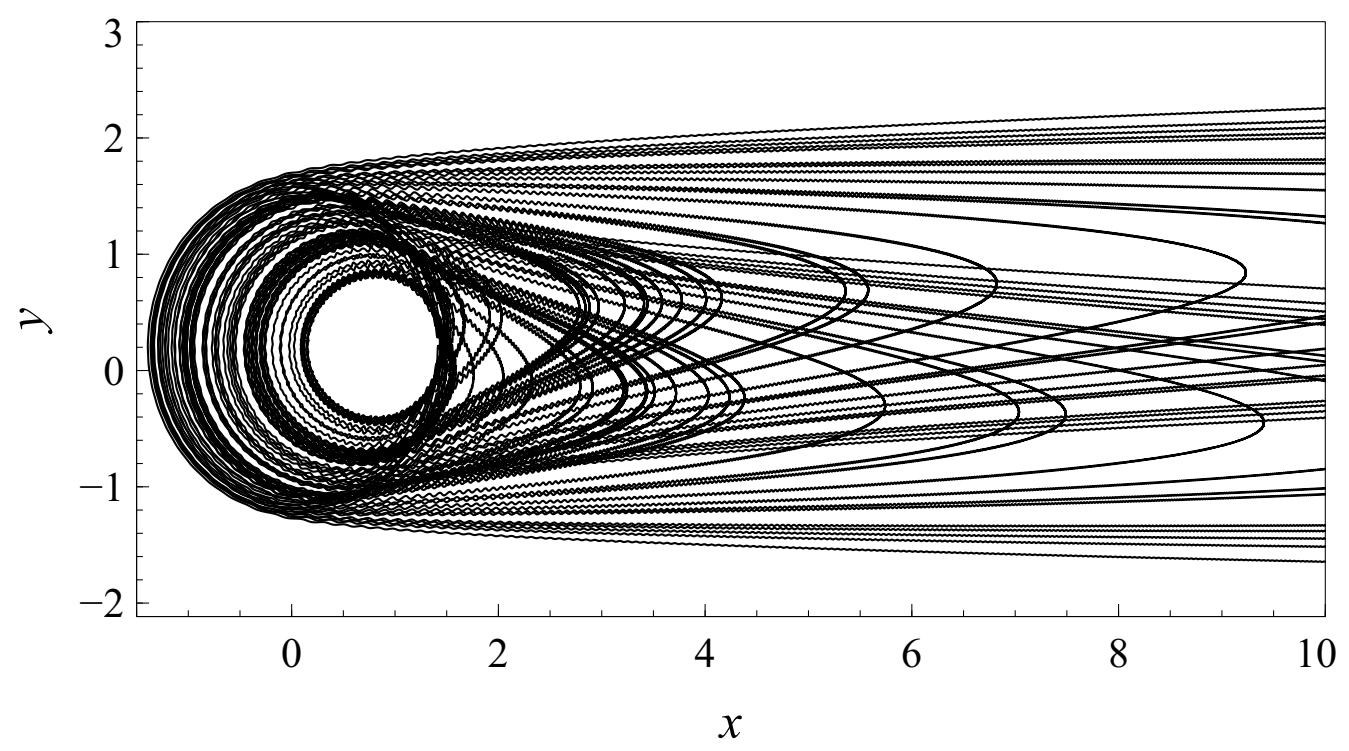

Figure 13. Long term dynamics in the slow plane. 


\section{Passage through a resonance}

\subsection{Description of passage through a resonance}

Consider again the slow-fast Hamiltonian system (1.2) with arbitrary numbers of degrees of freedom for fast and slow variables. Assume that the fast system is integrable. Let $I, \varphi$ be the action-angle variables for this system, $I \in \mathbb{R}^{n}, \varphi \in \mathbb{T}^{n}$ modd $2 \pi$. Let $H_{0}(I, y, x)$ be the Hamiltonian $E$ expressed via $I, y, x$. By averaging of the equations of motion over $\varphi$, we obtain the equations of adiabatic approximation of form (1.3) similarly to Section 1.2. The vector of the unperturbed frequencies of the fast motion is

$$
\omega(I, y, x)=\frac{\partial H_{0}(I, y, x)}{\partial I} .
$$

Even if $I$ remains constant, as the adiabatic approximation predicts, the evolution of the slow variables $y, x$ would lead to an evolution of $\omega(I, y, x)$. The components of the vector $\omega$ change slowly, and resonant relations ${ }^{2}$ between them appear and disappear. Resonances could be obstacles to validity of the averaging method in this case. Averaging over $\varphi$ is averaging over the torus $\mathbb{T}^{n}$, while in the case of a resonance the trajectory may even not visit some part of $\mathbb{T}^{n}$. Consider the two-frequency case: $n=2, \omega=\left(\omega_{1}, \omega_{2}\right), \varphi=\left(\varphi_{1}, \varphi_{2}\right)$. In the plane with coordinates $\omega_{1}, \omega_{2}$ the resonances are represented by the straight lines with rational slopes passing through the coordinate origin: $k_{1} \omega_{1}+k_{2} \omega_{2}=0,\left(k_{1}, k_{2}\right) \in \mathbb{Z}^{2} \backslash\{0\}$, with co-prime $k_{1}, k_{2}$. This simple structure of resonances allows to study effects of each resonance separately if some general enough conditions are satisfied. This was first used by P.A.M. Dirac to show that under some conditions the value $I$ is approximately conserved, i.e. $I$ is an adiabatic invariant [18], and by V.I. Arnold to obtain estimates of accuracy of the averaging method [3]. Principal phenomena associated with the effect of a single resonance are capture into resonance and scattering on resonance.

Let $I(t), y(t), x(t), \varphi(t)$ be a solution of the exact system (1.2) expressed in the variables $I, y, x, \varphi$. Consider a resonant surface $k_{1} \omega_{1}(I, y, x)+k_{2} \omega_{2}(I, y, x)=0$ in the space of slow variables $I, y, x$.

Capture into resonance can be described as follows. The point $(I(t), y(t), x(t))$ in the space of the slow variables moves in line with the prediction of the adiabatic approximation up to arrival to the resonant surface. Then it suddenly starts to move along the resonant surface in its $O(\sqrt{\varepsilon})$-neighbourhood (Fig. 14). This regime of motion continues for (at least) time of order $1 / \varepsilon$. Then the point $(I(t), y(t), x(t))$ may leave the neighbourhood of the resonant surface. In this case it continues its motion in line with the prediction of the adiabatic approximation with a new initial condition. Possibility of such a scenario was indicated in [17]. Capture into resonance was first considered in details in [22] in study of capture of Mercury

\footnotetext{
${ }^{2}$ For a vector $\omega \in \mathbb{R}^{n}$ a resonant relation is any relation of the form $\omega \cdot k=0$ with $k \in \mathbb{Z}^{n} \backslash\{0\}$. Here "." denotes the standard Euclidian dot product.
} 


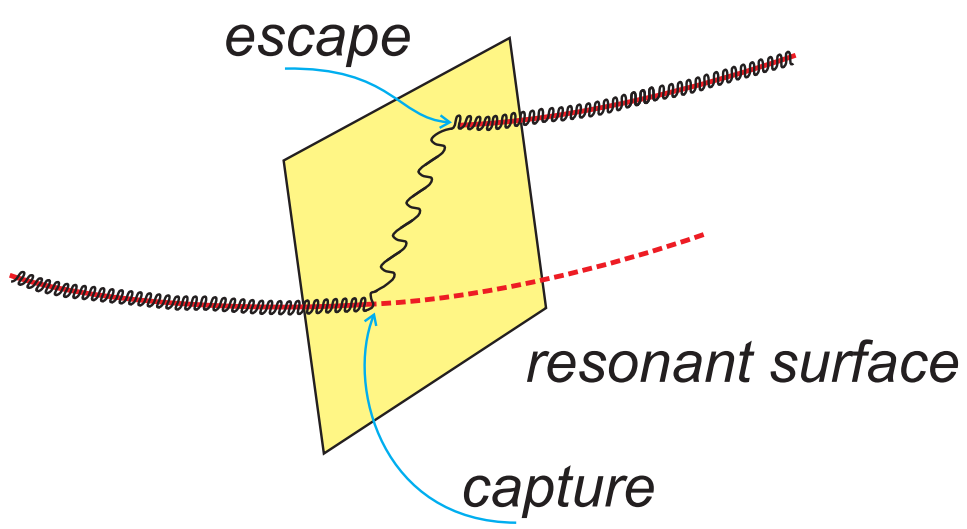

Figure 14. Capture into a resonance and escape from it.

into spin-orbital resonance due to a tidal dissipation. There a probabilistic interpretation of this phenomenon was suggested (it is similar to the probabilistic approach to separatrix crossing discussed in Sect. 2.1). The probability of capture for one passage through a resonance is small, $O(\sqrt{\varepsilon})$. There is a formula for this probability [29]. The dynamics of captured phase points can be approximately described by averaging along trajectories of an auxiliary system (pendulum-like system). Solution of the obtained averages system allows to construct an in-out (or entrance-exit) function which is a relation between the positions of capture into the resonance and escape from the resonance [29].

Scattering on resonance can be described as follows (in this description we consider an isolated resonance). The point $(I(t), y(t), x(t))$ in the space of the slow variables approaches the resonant surface, crosses it, and then moves off the resonant surface. Far from the resonant surface, the point $(I(t), y(t), x(t))$ oscillates with an amplitude $O(\varepsilon)$ near some trajectory of the adiabatic approximation system. However, these trajectories are different for motions towards and off the resonant surface. The mismatch of these two trajectories at the resonant surface is a value of order $O(\sqrt{\varepsilon})$, Fig. 15. This value is interpreted as a random scattering on resonance because it has a sensitive dependence on initial data (similarly to the dynamical jump of adiabatic invariant in Sec. 2.1). Scattering on resonance was first considered in [17], where a formula for the value of scattering is provided.

Both in cases of capture into resonance and passage through resonance without capture, the resonant phase $\gamma=k_{1} \varphi_{1}+k_{2} \varphi_{2}$ rotates in one direction when the phase point moves towards the resonant surface, and rotates in the direction which is opposite to the initial one, when the phase point moves off the resonant surface. In the case of capture into resonance, the resonant phase makes many oscillations in the process of motion along the resonant surface. In the case of passage through resonance without capture, the resonant phase does not oscillate.

Study of effects of a single resonance in two-frequency system can be reduced to study of a resonance in one-frequency system. For this reduction one can make a canonical transfor- 


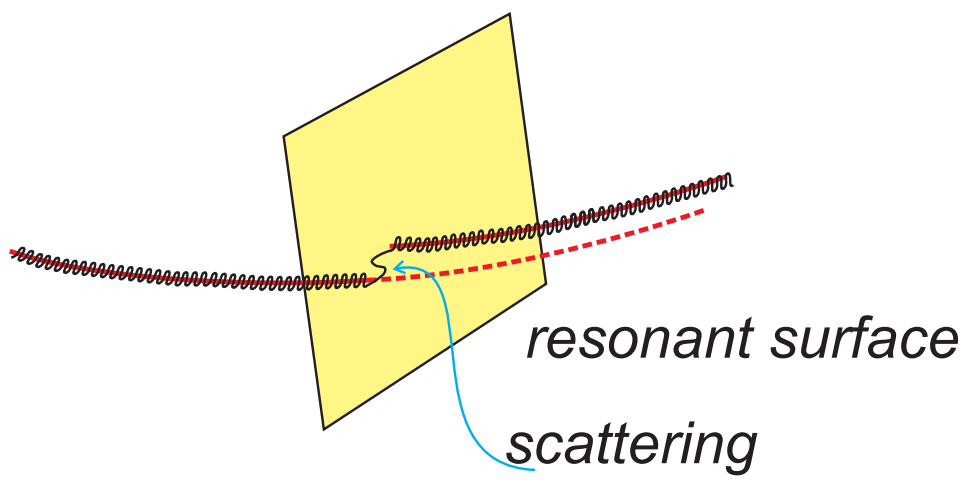

Figure 15. Scattering on a resonance.

mation of variables to introduce new angles $\gamma=k_{1} \varphi_{1}+k_{2} \varphi_{2}, \chi=l_{1} \varphi_{1}+l_{2} \varphi_{2}$ with integer $l_{1}, l_{2}$ such that $k_{1} l_{2}-k_{2} l_{1}=1$. Averaging of the Hamiltonian over the non-resonant phase $\chi$ gives a one-frequency system (the variable canonically conjugate to $\chi$ is a parameter of this system).

In view of the possibility of the described reduction, we will discuss below a one-frequency system: $n=1$. Assume, in addition, that $m=1$. Thus we have a slow-fast Hamiltonian system with two degrees of freedom. The resonant relation is $\omega(I, y, x)=0$. Consider 3 dimensional space of slow variables $x, y, I$ (Fig. 16). In the adiabatic approximation, the motion takes place in the plane $I=$ const. In this plane we have a Hamiltonian system with one degree of freedom for the variables $y, x$. Assume that the trajectories of this system are closed curves. Assume that the resonant surface $\{\omega(I, y, x)=0\}$ crosses the considered plane $I=$ const. In the adiabatic approximation the motion is periodic, phase points cross the resonant surface repeatedly.

Consider the motion of phase points with initial conditions in some ball. Adiabatic approximation predicts that, during time of order $1 / \varepsilon$, these phase points will cross the resonant surface several $(\sim 1)$ times. For this time interval, a fraction $\sim \sqrt{\varepsilon}$ (in the sense of measure) of the initial ball may correspond to motion with capture into resonance (the probability of capture into resonance is a value $\sim \sqrt{\varepsilon}$ ). For these initial conditions the adiabatic invariance is destroyed. For the remaining initial conditions there is a deviation $\sim \sqrt{\varepsilon}$ from predictions of the adiabatic approximation due to scattering on the resonance. (See the first column of the table in Fig. 17.)

For a time interval $\sim 1 / \varepsilon^{3 / 2}$, a subset of measure $\sim 1$ of the considered ball of initial conditions may correspond to motion with capture into resonance. Such an example is constructed in [28]. Non-zero mean value of scattering also plays the principal role in dynamics on this time interval. It produces a deterministic drift along the resonant surface at a distance $\sim 1$ [19] (repulsion from resonance, [19]). (See the second column of the table in Fig. 17.) Thus, on such a time interval adiabatic invariance is completely destroyed. 


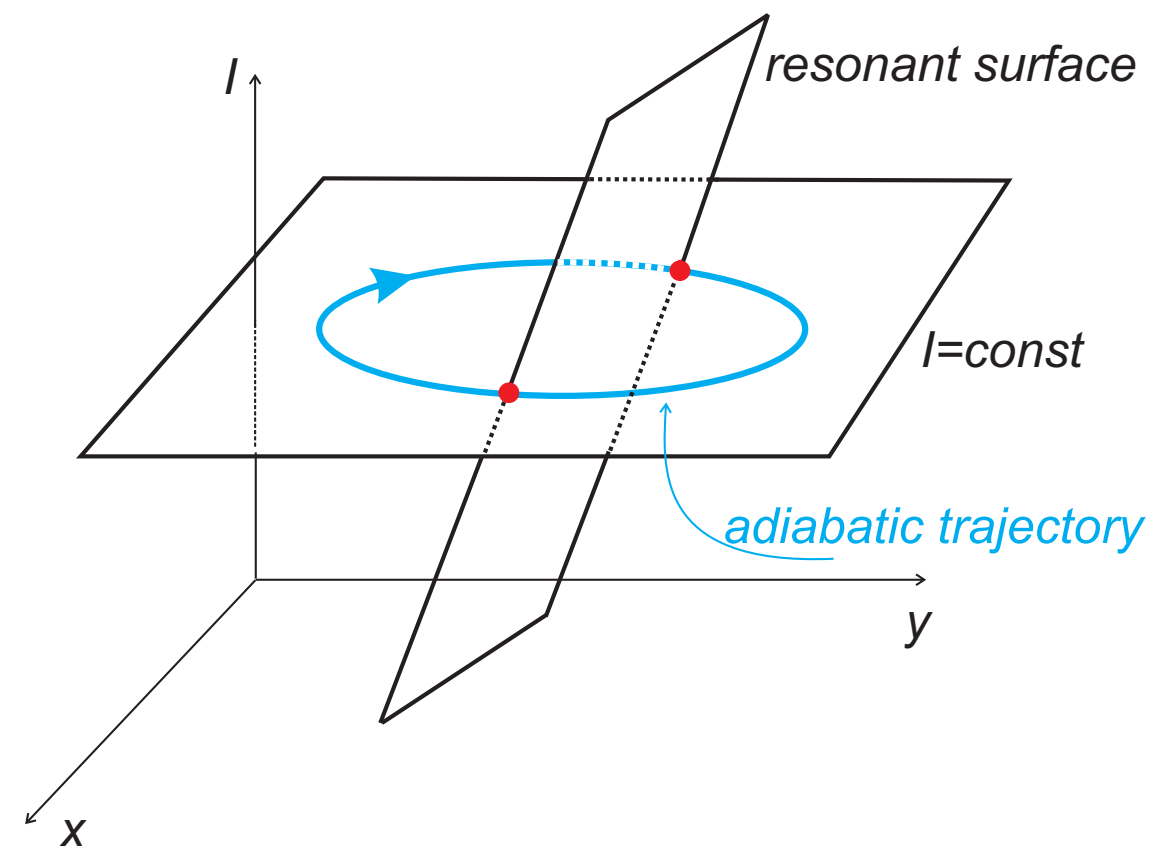

Figure 16. Adiabatic trajectory and resonant surface.

\begin{tabular}{|l|l|l|l|}
\hline time & $1 / \varepsilon$ & $1 / \varepsilon^{3 / 2}$ & $1 / \varepsilon^{2}$ \\
\hline mes(capture) & $\cong \varepsilon^{1 / 2}$ & $\cong 1$ & $\cong 1$ \\
\hline $\begin{array}{l}\text { average } \\
\text { scattering }\end{array}$ & $\cong \varepsilon^{1 / 2}$ & $\cong 1$ (drift [19]) & $\cong 1$ \\
\hline $\begin{array}{l}\text { standard } \\
\text { deviation of } \\
\text { scattering }\end{array}$ & $\cong \varepsilon^{1 / 2}$ & $\cong \varepsilon^{1 / 4}$ & $\begin{array}{l}\cong 1 \\
\text { (diffusion) }\end{array}$ \\
\hline
\end{tabular}

Figure 17. Principal time scales. 
There is no rigorous theory for dynamics on time interval $\sim 1 / \varepsilon^{2}$. It seems that the deviation of the scattering from its mean value produces a diffusive change of order 1 in the value of action $I$. (See the third column of the table in Fig. 17; we assume that during this time the phase points do not leave a compact domain.)

Heuristically, it is possible to consider the results of consecutive crossings of a resonance as independent random values for the following reason. The change of the action $I$ due to scattering on resonance depends on the phase $\varphi$ at the moment of crossing the resonant surface. Consider two phase points with a small difference of phases $\delta \varphi$ that cross the resonant surface; values of all other variables are the same. After crossing the resonance, the values of action for these two points differ by a value $\delta I \sim \varepsilon^{1 / 2} \delta \varphi$ due to scattering on resonance. Thus, after this scattering the phases of these points rotate with different frequencies. The difference of these frequencies is $\delta \omega \sim \delta I \sim \varepsilon^{1 / 2} \delta \varphi$. Motion till the next crossing of the resonance takes time $T \sim 1 / \varepsilon$. During this time, the difference in phases grows to a value $\delta \varphi^{\prime} \sim T \delta \omega \sim \varepsilon^{-1 / 2} \delta \varphi \gg \delta \varphi$. This stretching of phase indicates that the values of phases at consecutive crossings of resonances are independent. Proof of the independence in the one-frequency case is an intermediate result in [19].

Numerics shows that the evolution of the distribution function of phase points due to many captures, escapes and scatterings on a resonance can be described by a non-local PDE of the Fokker-Planck type [7].

\subsection{Example: dynamics of a charged particle under the action of a constant magnetic field and an electromagnetic wave in plasma $[40]$}

\subsubsection{Model}

Consider the motion of a charged particle acted upon by a constant background magnetic field and a high-frequency electromagnetic harmonic wave that propagates in plasma at an angle to this magnetic field. Averaging over the phase of the wave washes out the influence of the wave. Thus, the motion in the averaged system is a motion in a constant magnetic field. It is a helical motion which is composed of the rotation in the Larmor circle perpendicular to the magnetic field and the drift of this circle along the magnetic field. In the process of Larmor rotation the projection of the particle velocity onto the direction of wave propagation changes. At some moment of time this projection may be equal to the phase velocity of the wave ${ }^{3}$. This is a particular case of passage through a resonance discussed in the previous Section. The phase of the wave at the position of the particle is the fast angular variable. Its frequency vanishes at the resonance. The slow motion in the adiabatic approximation

\footnotetext{
${ }^{3}$ Recall that this is an electromagnetic wave in a plasma, not in a vacuum. Its phase velocity could be much smaller than the speed of light.
} 


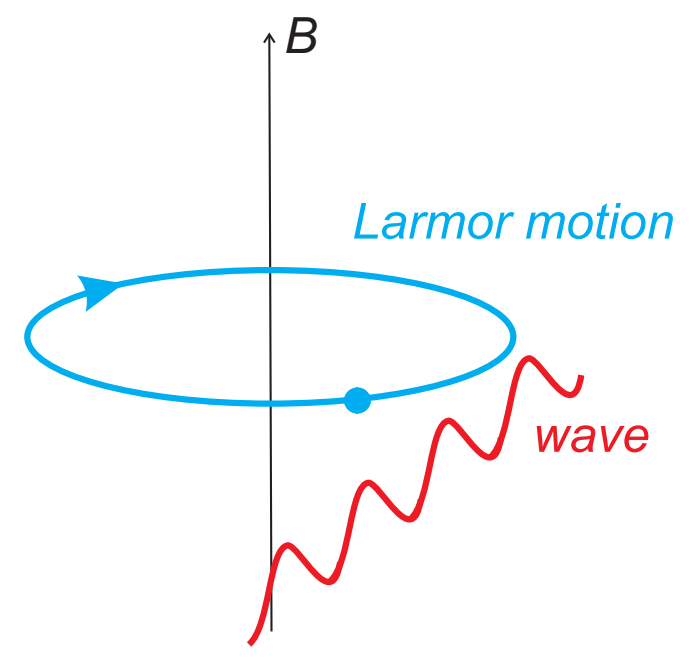

Figure 18. A charged particle acted upon by a constant background magnetic field and a high-frequency electromagnetic wave.

is the motion in the constant magnetic field. Capture and scattering may take place at the resonance.

\subsubsection{Equations of motion}

Introduce a Cartesian coordinate system $O x y z$ in which the background magnetic field is directed along $O z$, and the wave propagates along some direction in Oyz plane, Fig. 19. We assume that the field of the wave has the linear polarisation: its electric component is directed along the axis $O x$, while its magnetic component is perpendicular to it and to the direction of the wave propagation. Denote $\mathbf{B}_{\mathbf{0}}=\left(0,0, B_{0}\right), \mathbf{B}=\left(0, B_{y}, B_{z}\right), \mathbf{E}=\left(E_{x}, 0,0\right)$ vectors of the background magnetic field, of the magnetic field of the wave, and of the electric field of the wave, respectively. Denote $\mathbf{k}=\left(0, k_{2}, k_{3}\right), \omega, \varphi=k_{2} y+k_{3} z-\omega t$ the wave vector, the frequency, and the phase of the wave. Then

$$
B_{y}=\frac{B k_{3}}{k} \sin \varphi, B_{z}=-\frac{B k_{2}}{k} \sin \varphi, E_{x}=\frac{B \omega}{k c} \sin \varphi
$$

Here $B=\sqrt{B_{y}^{2}+B_{z}^{2}}, k=\sqrt{k_{2}^{2}+k_{3}^{2}}, c$ is the speed of light. Vectors $\mathbf{B}$ and $\mathbf{E}$ are related according to Maxwell's equations: $\operatorname{div} \mathbf{B}=0, \operatorname{curl} \mathbf{E}=-\frac{1}{c} \frac{\partial \mathbf{B}}{\partial t}$.

Introduce a vector potential $\mathbf{A}$ for the magnetic field $\left(\mathbf{B}_{\mathbf{0}}+\mathbf{B}=\operatorname{curl} \mathbf{A}\right)$. We have

$$
\mathbf{A}=\left(-B_{0} y-\frac{B}{k} \cos \left(k_{2} y+k_{3} z-\omega t\right), 0,0\right)
$$

Dynamics is described by the Hamiltonian system with Hamilton's function

$$
H=\frac{1}{2 m}\left(\mathbf{p}-\frac{e}{c} \mathbf{A}\right)^{2}
$$




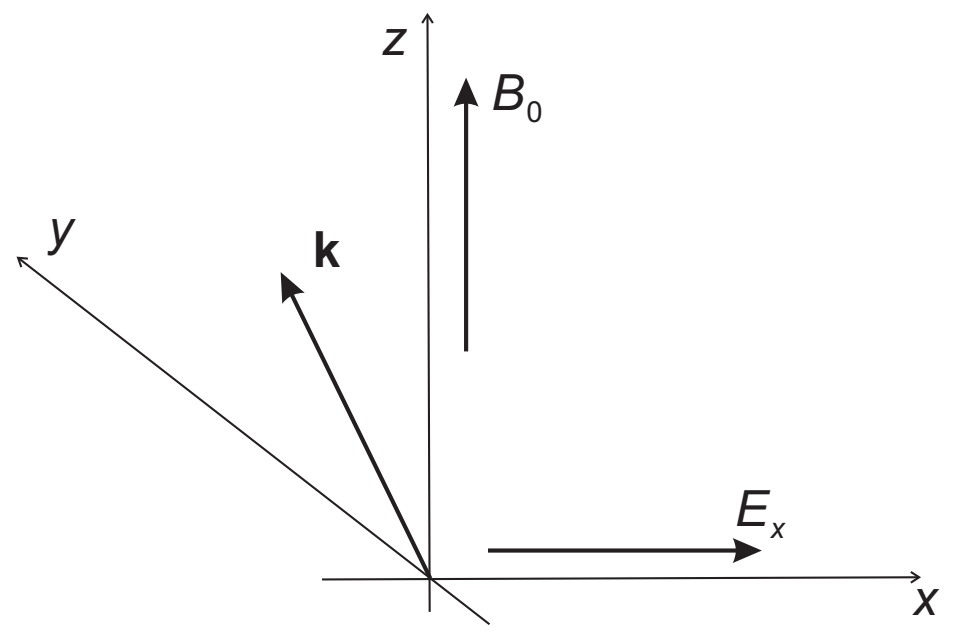

Figure 19. Configuration of fields.

and conjugate variables $\mathbf{p}=\left(p_{x}, p_{y}, p_{z}\right), \mathbf{q}=(x, y, z)$. Here $m$ and $e$ are the mass and charge of the particle.

The considered system has $3 \frac{1}{2}$ degrees of freedom. The Hamiltonian does not depend on $x$. Thus $p_{x}=$ const. Without loss of generality we put $p_{x}=0$, this is achieved by choosing an origin of $y$-coordinate. We are left with $2 \frac{1}{2}$ degrees of freedom.

After some rescaling of variables, time, and parameters, the Hamiltonian for dimensionless variables takes the form (see details in [40])

$$
H=\frac{1}{2} p_{y}^{2}+\frac{1}{2} p_{z}^{2}+\frac{1}{2}\left(y+\varepsilon \cos \left(\varepsilon^{-1}\left(k_{2} y+k_{3} z-\omega t\right)\right)^{2} .\right.
$$

Here $\varepsilon=\frac{1}{k R_{0}}$, where $R_{0}$ is a typical Larmor radius of motion in the field $\mathbf{B}_{\mathbf{0}}$. We assume that $0<\varepsilon \ll 1$. Canonically conjugate pairs of variables are $y, p_{y}$ and $z, p_{z}$.

Introduce $\varphi=\varepsilon^{-1}\left(k_{2} y+k_{3} z-\omega t\right)$ as a new variable. To this end we make a canonical transformation of variables $\left(p_{y}, p_{z}, y, z\right) \mapsto(\varepsilon I, p, \varphi, q)$ with the generating function $W=$ $I\left(k_{2} y+k_{3} z-\omega t\right)+p y$. The new variables are

$$
\varphi=\varepsilon^{-1}\left(k_{2} y+k_{3} z-\omega t\right), q=y, I=p_{z} / k_{3}, p=p_{y}-p_{z} k_{2} / k_{3} .
$$

The new Hamiltonian is

$$
H=H_{0}+\varepsilon H_{1}=-\omega I+\frac{1}{2}\left[\left(p+I k_{2}\right)^{2}+\left(I k_{3}\right)^{2}+q^{2}\right]+\varepsilon q \cos \varphi+\frac{1}{2} \varepsilon^{2} \cos ^{2} \varphi .
$$

We have $H=$ const in the process of motion. Thus, in the principal approximation the motion takes place on the surface

$$
H_{0}=H_{0}(I, p, q)=-\omega I+\frac{1}{2}\left[\left(p+I k_{2}\right)^{2}+\left(I k_{3}\right)^{2}+q^{2}\right]=\text { const. }
$$


Introduce also the new time $\tilde{t}=t / \varepsilon$. The system has now two degrees of freedom with pairs of conjugate variables $(\varphi, I)$ and $\left(\varepsilon^{-1} q, p\right)$. Equations of motion have the standard slow-fast form described in Sect. 3.1:

$$
\begin{aligned}
\dot{\varphi} & =-\omega+k_{2} p+k^{2} I, \\
\dot{I} & =\varepsilon q \sin \varphi+\varepsilon^{2} \sin \varphi \cos \varphi \\
\dot{q} & =\varepsilon\left(p+I k_{2}\right), \\
\dot{p} & =-\varepsilon q-\varepsilon^{2} \cos \varphi
\end{aligned}
$$

with the fast phase $\varphi$ and the slow variables $I, q, p$.

The averaged system has the Hamiltonian $H_{0}(I, p, q)$. The averaged equations

$$
\begin{aligned}
\dot{I} & =0, \\
\dot{q} & =\varepsilon\left(p+I k_{2}\right), \\
\dot{p} & =-\varepsilon q
\end{aligned}
$$

describe Larmor rotation in the background magnetic field.

\subsubsection{Resonant phenomena}

The trajectories of the averaged system in the $q, p$-plane are the circles $\left(p+I k_{2}\right)^{2}+q^{2}=$ const. The resonance is represented by the straight line $p=\left(\omega-k^{2} I\right) / k_{2}$. Intersections of these circles and straight line lead to resonant phenomena.

In the case of capture into resonance, the dynamics of $p, q$ is described by the Hamiltonian system with the Hamiltonian

$$
\Lambda(p, q)=H_{0}\left(I_{R}(p), p, q\right)=\frac{1}{2} q^{2}+\frac{k_{3}^{2}}{2 k^{2}} p^{2}+\frac{\omega k_{2}}{k^{2}} p+\text { const }
$$

where $I=I_{R}(p)=\left(\omega-k_{2} p\right) / k^{2}$ is the equation of the resonant surface in $I, q$, $p$-space. Thus, in the case of capture into resonance the system switches from the trajectory of the Hamiltonian $H_{0}(I, p, q), I=$ const to a trajectory of the Hamiltonian $\Lambda(p, q)$. The captured particle leaves a neighbourhood of the Larmor trajectory $I=$ const and moves in such a way that the resonant condition $I=\left(\omega-k_{2} p\right) / k^{2}$ is kept.

One can show that capture into resonance is possible under the condition which in the original variables has the form $\left(k_{2} B_{0}\right) /(k B)<1[40]$. In the $q, p$-plane the resonant line $p=p_{R}=\left(\omega-k^{2} I\right) / k_{2}$ may cross the averaged trajectory $\left(p+I k_{2}\right)^{2}+q^{2}=$ const at two points. Of these points, capture is possible at the point where $|q|$ grows along the averaged trajectory [40]. A phase point captured at $q=q_{*}, p=p_{*}$ will escape from the resonance when $q$ returns to the value $q_{*}$ in the captured motion, i.e. when $p=p_{* *}$ such that $\Lambda\left(p_{* *}, q_{*}\right)=\Lambda\left(p_{*}, q_{*}\right)$. Thus, $p_{* *}=-p_{*}-2 \omega k_{2} / k_{3}^{2}$. 


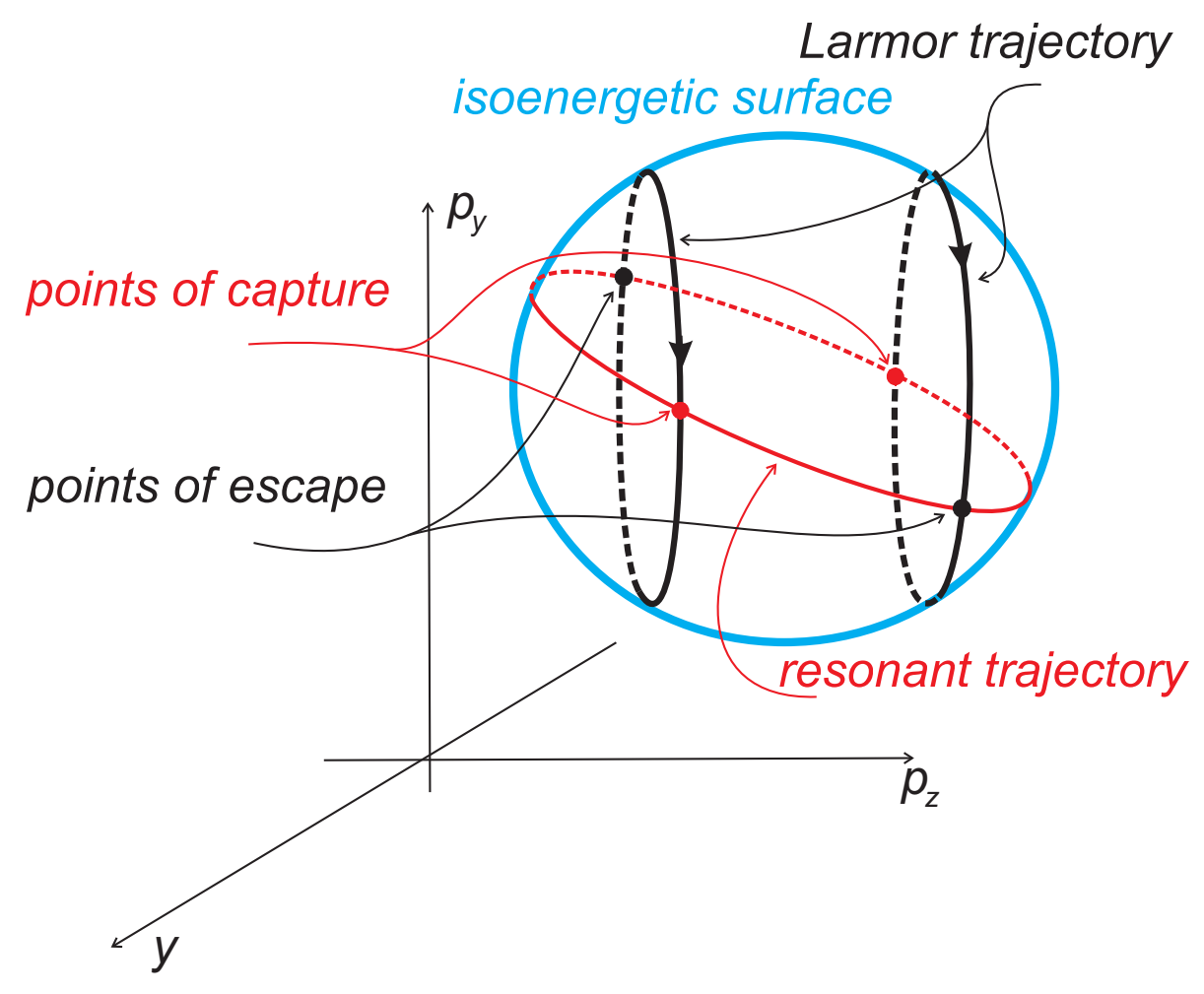

Figure 20. Schematic description of dynamics with captures into resonance.

In the $\left(y, p_{z}, p_{y}\right)$-space dynamics in the principal approximation schematically looks as follows. The motion takes place on the iso-energetic surface, which is a sphere $\frac{1}{2}\left(y^{2}+p_{y}^{2}+p_{z}^{2}\right)-$ $\omega p_{z} / k_{3}=$ const (Fig. 20). Adiabatic (Larmor) trajectories are the circles which are sections of this sphere by the planes $p_{z}=$ const. The resonant surface is the plane $\omega-k_{2} p_{y}-k_{3} p_{z}=0$ passing through the centre of this sphere. Trajectories of captured particles are arcs of the circle which is the section of the iso-energetic sphere by the resonant plane (Fig. 20). A particle makes many Larmor rounds and then is suddenly captured into resonance. It moves in the resonance and is released from it when the resonant trajectory intersects the Larmor circle symmetric to the original one with respect to the centre of the iso-energetic sphere. Then the particle moves in this new Larmor circle and is suddenly captured into resonance after many Larmor rounds. It moves in the resonance and is released from it at the original Larmor circle, etc. This description, however, is valid in the principal approximation only. Larmor rounds do not repeat each other exactly due to scattering on the resonance. A trajectory with two captures and two escapes obtained numerically is shown in Fig. 21. If captures are not possible, $\left(k_{2} B_{0}\right) /(k B)>1$, adiabatic invariance is still destroyed due to scatterings on the resonance. This is illustrated in Fig. 22 where the return map with a period $2 \pi / \omega$ is shown for a long phase trajectory with multiple scatterings on the resonance (see further details in [40]). 


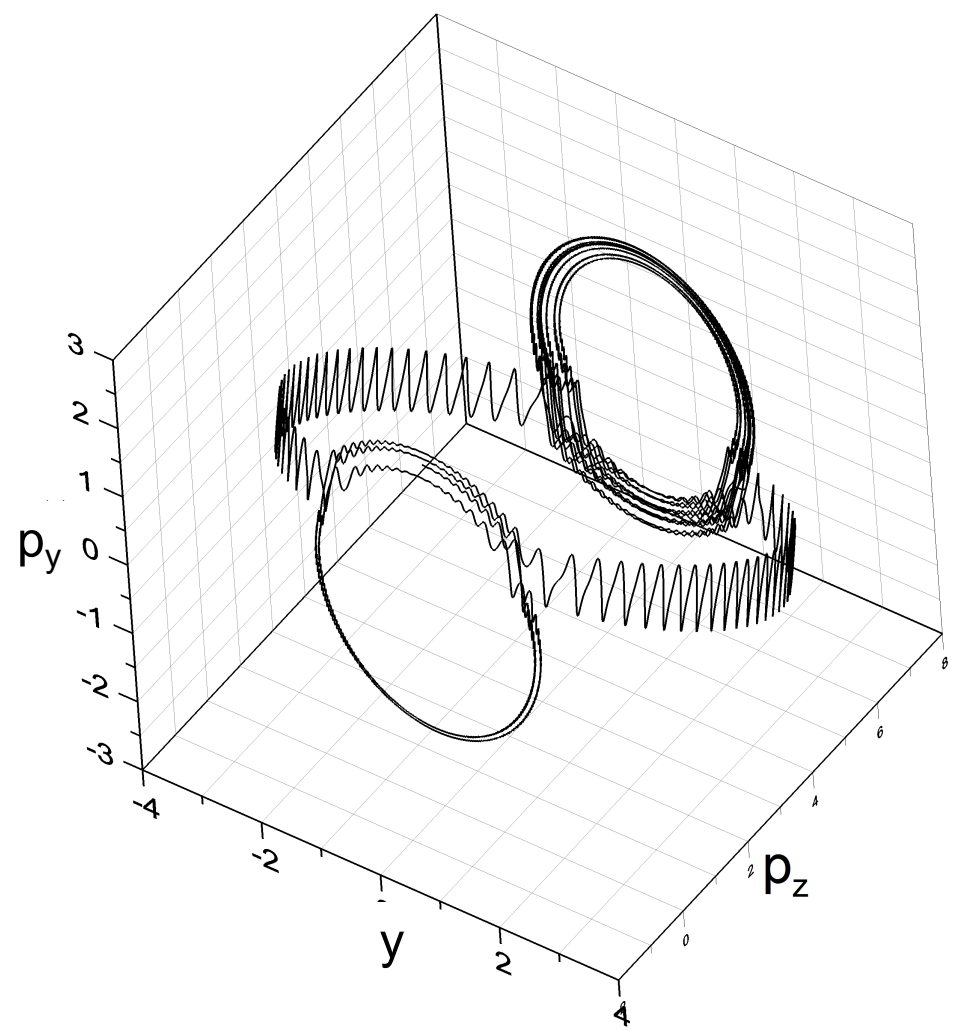

Figure 21. Captures into resonance and escapes from resonance. 


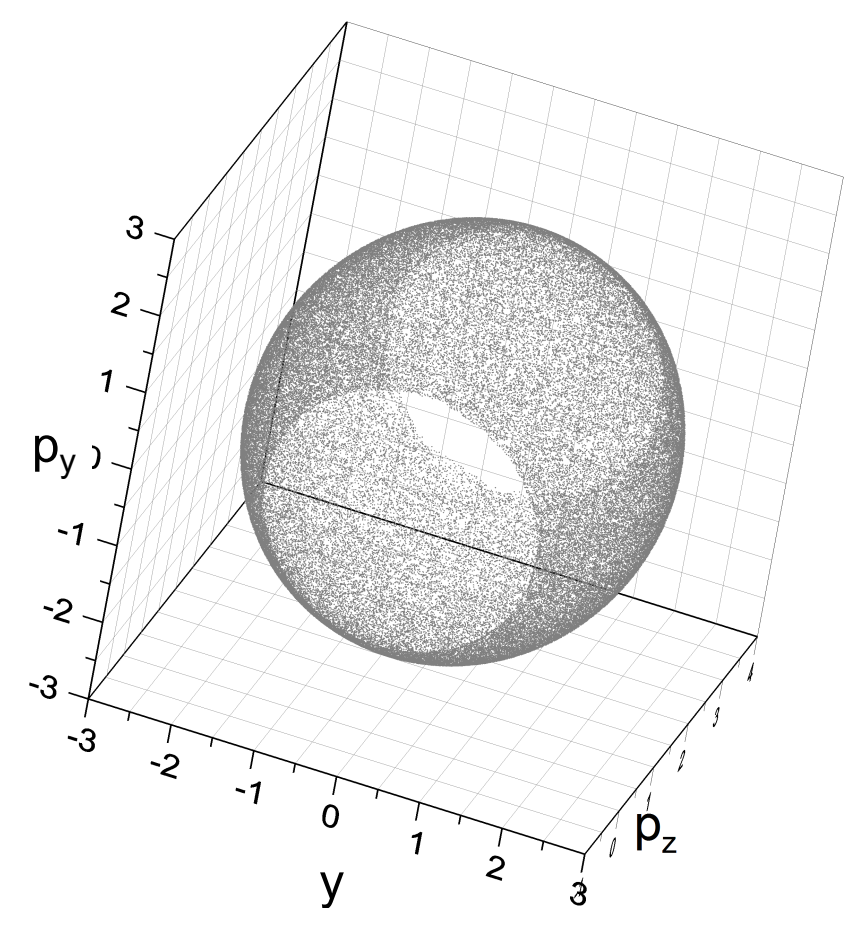

Figure 22. Effects of scatterings on resonance.

\section{Change of the mode of motion in systems with col- lisions}

Change of the mode of motion from motion with collisions to motion without collisions may lead to destruction of adiabatic invariance. It is shown in [31] for the motion of a charged particle in a billiard in a strong nonuniform magnetic field. This is a usual planar mathematical billiard with a perfectly reflecting smooth closed boundary, but the particle in the billiard has a charge, and there is a magnetic field perpendicular to the plane of the billiard.

In the case of a strong uniform magnetic field there are two modes of motion: Larmor rotation without collisions with the boundary and skipping along the boundary due to collisions with it, Fig. 23, (a). There is no switching between these two modes. The skipping motion has an adiabatic invariant. Namely, in this motion the particle moves in arcs of Larmor circles. Take the distance of the centre of such a circle from the boundary and divide it by the strength of the magnetic field to have a value $\sim 1$. This value is an adiabatic invariant: it remains about constant for a long time interval that includes many collisions [37]. Moreover, if the boundary of the billiard is a smooth enough curve, then this value is a perpetual adiabatic invariant [10].

We are interested in the case of a strong nonuniform magnetic field of strength $B(x, y) / \varepsilon, B(x, y)>$ 

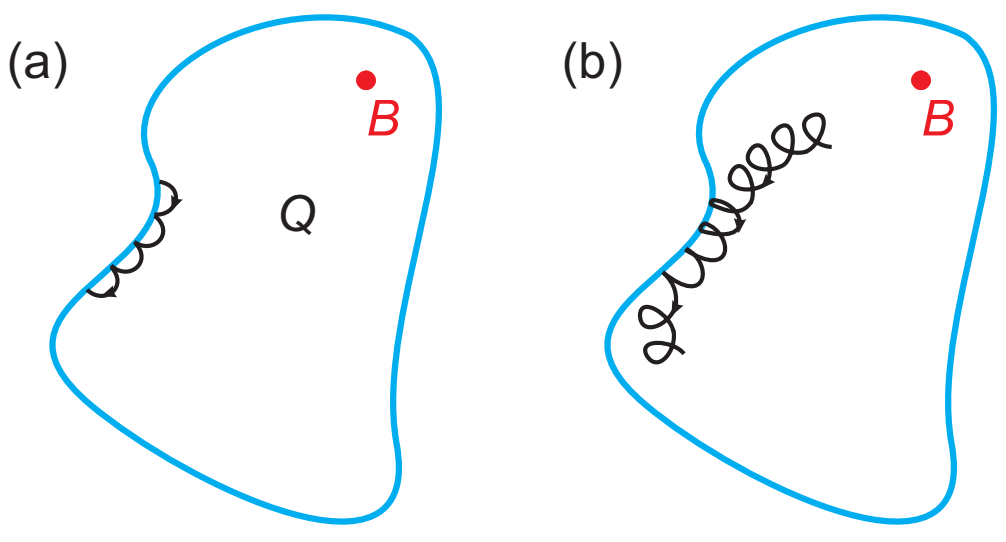

Figure 23. (a) Modes of particle motion for a billiard with an uniform magnetic field. (b) Switching between modes of motion for a billiard with a nonuniform magnetic field.

const $>0$, where $(x, y)$ are the Cartesian coordinates in the plane of the billiard, $\varepsilon$ is a small parameter of the problem. Let at some moment of time a particle be far enough from the boundary of the billiard. The motion of the particle can be represented as a composition of the motion in a Larmor circle and a slow drift of the centre of this circle along the line $B(x, y)=$ const (a gradient drift [36]). The flux of the magnetic field through the Larmor circle divided by $\varepsilon$ is an adiabatic invariant of this motion. ${ }^{4}$ The speed of the gradient drift is $\sim \varepsilon$. In the process of the gradient drift the particle may approach the boundary of the billiard and collide with it. Then the mode of motion switches to skipping along the boundary due to collisions with it, Fig. 23, (b). This motion also has an adiabatic invariant. Namely, for this motion the particle moves approximately on arcs of Larmor circles. Consider a small domain ( $a$ cell) bounded by such an arc and a segment of the boundary of the billiard. Then the flux of the magnetic field through this domain divided by $\varepsilon$ is an adiabatic invariant [31]. Because the step of skipping is small, of order $\varepsilon$, this skipping is considered in the principal approximation as a drift along the boundary of the billiard. This drift is described by a Hamiltonian system, the adiabatic invariant is a first integral of this system [31]. The speed of this drift is $\sim 1$. One can show that initially the particle drifts in the direction of decay of the value of $B(x, y)$ along the boundary of the billiard. The value of $B(x, y)$ along the boundary reaches its minimum during this drift and starts growing. When $B$ reaches its value at the start of the skipping with an accuracy $O(\varepsilon)$, the skipping motion switches back to the gradient drift. As the radius of the Larmor circle is small, $\sim \varepsilon$, the position of the particle can be identified in the principal approximation with the centre of this circle for the gradient drift and with the projection of this centre onto the boundary of the billiard for the skipping motion. Thus, in the adiabatic approximation the particle moves in a closed trajectory. This trajectory consists of an arc of a curve $B(x, y)=$ const with the endpoints on the boundary of the billiard, and an arc of the boundary of the billiard between these

\footnotetext{
${ }^{4}$ Thus, we can say that the radius of this circle or the area of this circle is an adiabatic invariant, but it is more convenient to talk about the flux of the magnetic field in this context. This adiabatic invariant is proportional to the magnetic moment of the particle [36].
} 
points. On the latter arc the value of $B$ is less than at the endpoints.

This is, however, a description in the adiabatic approximation. It turns out that small jumps of the adiabatic invariant at switching between the modes of motion destroy this description over a long enough time. This can be explained as follows. The adiabatic invariant, as it is defined above via area, remains a continuous function of the radius of the Larmor circle and the position of its centre at the switching. Indeed, at the switching the Larmor circle is tangent to the boundary of the billiard, and a cell is the domain bounded by this Larmor circle. The value of this adiabatic invariant along the particle trajectory is conserved with an accuracy $O(\varepsilon)$ on time intervals of length $\sim 1 / \varepsilon$. For motion far from the switching points one can introduce an improved adiabatic invariant, which is conserved with an accuracy $O\left(\varepsilon^{2}\right)$. However, this adiabatic invariant undergoes a change (a jump) $\sim \varepsilon^{3 / 2}$ at passing through a narrow neighbourhood of the switching point. There is an asymptotic formula for this jump [6]. The value of this jump depends on the position of the particle on the Larmor circle (a phase) at the moment when this circle touches the boundary of the billiard. This phase can be treated as a random value, because the Larmor rotation is fast (the angular speed $\sim 1 / \varepsilon)$. The total jump due to switching from the gradient drift to drift along the boundary and back can be considered as a random value with the average 0. Assuming independence of these random values (see below), we conclude that accumulation of these jumps should destroy adiabatic invariance during time intervals $\sim 1 / \varepsilon^{4}$. This independence can be heuristically explained similarly to the case of passages through resonances as follows. Consider two particles with a small difference of phases $\delta \varphi$ at the same Larmor circle. After two switchings of modes of motion, the values of the adiabatic invariant for these two particles differ by a value $\delta I \sim \varepsilon^{3 / 2} \delta \varphi$. The speed of the gradient drift is proportional to the value of the adiabatic invariant. Thus, time of the gradient drift for these two particles differs by $T \sim \varepsilon^{-1} \delta I \sim \varepsilon^{1 / 2} \delta \varphi$. During this time, the difference in phase grows to a value $\delta \varphi^{\prime} \sim \varepsilon^{-1} T \sim \varepsilon^{-1 / 2} \delta \varphi \gg \delta \varphi$ (we use here that the Larmor frequency $\sim 1 / \varepsilon$ ). This stretching of the phase indicates that the values of the phases at two consecutive switches from the gradient drift to the drift along the boundary are independent.

If there are no switchings of the modes of motion, then the adiabatic invariance is conserved eternally.

These phenomena are illustrated in Fig. 24 (see Fig. 3 in [31]). In this example the boundary of the billiard is the circle $(x-1.5)^{2}+y^{2}=1.5^{2}$ in the $x, y$-plane. The magnetic field strength is $\varepsilon^{-1} B(x, y)$ with $B(x, y)=1+(x-0.5)^{2}+y^{2}, \varepsilon=0.1$. The charge and mass of the particle as well as the speed of light are set equal to 1 . The kinetic energy of the particle is constant: $\dot{x}^{2}+\dot{y}^{2}=1$.

Fig. 24, (a) presents a fragment of particle trajectory with an eternal skipping motion. The Poincaré return map for this trajectory for the section $y=0, \dot{y}<0$ is shown in Fig. 24, (c) in coordinates $x, \dot{x}$. Images of the return map form an invariant curve corresponding to the perpetual conservation of the adiabatic invariant. 

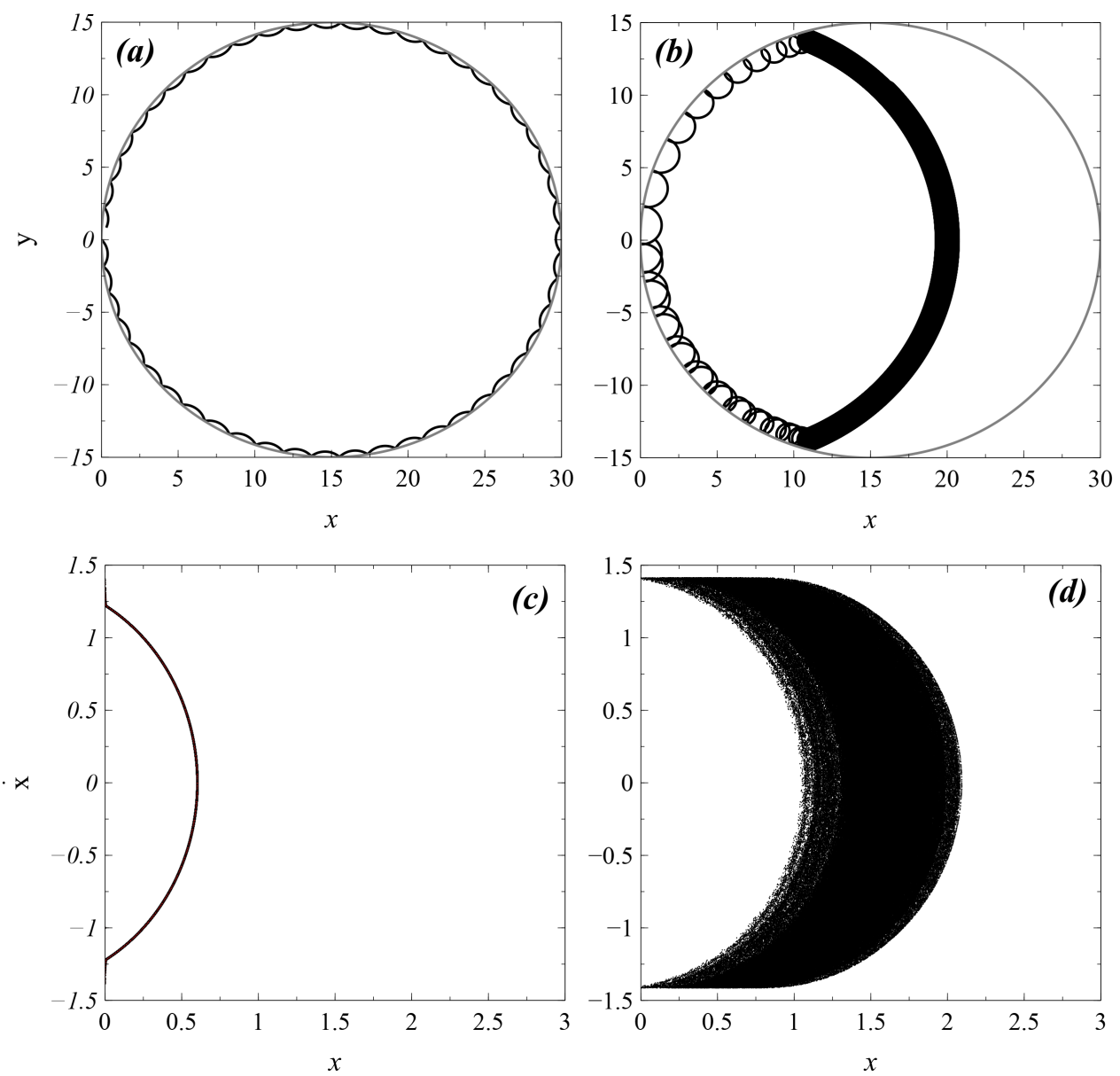

Figure 24. Fragments of two trajectories in a billiard in magnetic field and corresponding Poincaré sections. 
Fig. 24, (b) presents a fragment of particle trajectory with switchings between the modes of motion. For this trajectory, the evolution of the images of the Poincaré return map looks like the iterations on a slowly diffusing line of constancy of the adiabatic invariant, Fig. 24, (d). This diffusion destroys the adiabatic invariance.

\section{Conclusion}

In this review we have considered slow-fast Hamiltonian systems under an assumption that the fast system is integrable. Destruction of adiabatic invariance due to geometrical and dynamical jumps of adiabatic invariant at passage through a separatrix, due to capture into resonance and scattering on resonances, and due to jumps of an adiabatic invariant at change of mode of motion for systems with collisions was considered. It looks as these are the only known mechanisms of destruction of adiabatic invariance in the considered class of systems. In all cases, these mechanisms were related to switching between different regimes of motion in such systems.

Adiabatic invariance exists also in the cases when the fast system is not integrable [1, 24]. Destruction of adiabatic invariance there is considered, for different situations, in [38] and references therein, and in [21]. Mechanisms of this destruction are also associated with many switchings between different regimes of motion.

Destruction of adiabatic invariance in some volume-preserving systems is considered in [34] (see also references therein).

There is a close analogy between dynamics of slow-fast Hamiltonian systems and slow-fast symplectic maps. Results about destruction of adiabatic invariance for slow-fast symplectic maps due to passages through resonances can be derived from the corresponding results for slow-fast Hamiltonian systems (cf. Appendix in [29]).

\section{Acknowledgment}

This review is based on joint works with A V Artemyev, D Mourenas, V V Sidorenko, C Simo, D L Vainchtein, A A Vasiliev and L M Zelenyi. The author is deeply thankful to them. Additionally, the author is very thankful to A V Artemyev for numerous discussions and help in preparation of this review. The work was supported by the Leverhulme Thrust (grant RPG-2018-143). 


\section{References}

[1] Anosov D V 1960 Averaging in systems of ordinary differential equations with rapidly oscillating solutions Izv. Akad. Nauk SSSR, Ser. Mat. 24 721-742 (in Russian)

[2] Arnold V I 1963 Small denominators and problems of stability of motion in classical and celestial mechanics Russ. Math. Surv. 186 85-191

[3] Arnold V I 1965 Applicability conditions and an error bound for the averaging method for systems in the process of evolution through a resonance Soviet Math. Dokl. 6 331-334

[4] Arnold V I 1978 Mathematical Methods of Classical Mechanics (Graduate Texts in Mathematics vol 60 (New York: Springer) p x+462

[5] Arnold V I, Kozlov V V and Neishtadt A I 2006 Mathematical Aspects of Classical and Celestial Mechanics. Dynamical systems, III (Encyclopaedia of Mathematical Sciences) vol 3 (Berlin: Springer) p xiv +518

[6] Artemyev A V and Neishtadt A I 2015 Violation of adiabaticity in magnetic billiards due to separatrix crossings Chaos $\mathbf{2 5} 083109$

[7] Artemyev A V, Neishtadt A I, Vasiliev A A and Mourenas D 2016 Kinetic equation for nonlinear resonant wave-particle interaction Physics of Plasmas 23090701

[8] Artemyev A V, Neishtadt A I and Zelenyi L M 2013 Ion motion in the current sheet with sheared magnetic field - Part 1: Quasi-adiabatic theory Nonlinear Processes in Geophysics $20163-178$

[9] Artemyev A V, Neishtadt A I and Zelenyi L M 2014 Rapid geometrical chaotization in slow-fast Hamiltonian systems Physical Review E, 89 060902(R)

[10] Berglund N and Kunz H 1996 Integrability and ergodicity of classical billiards in a magnetic field J. Stat. Phys. 83 81-126

[11] Bogolyubov N N and Mitropolskij Yu A 1961 Asymptotic Methods in the Theory of NonLinear Oscillations (Delhi: Hindustan Publ. Corp.) p x+537

[12] Buc̈hner J and L. Zelenyi L M 1989 Regular and chaotic charged particle motion in magnetotaillike field reversals: 1. Basic theory of trapped motion Journal of Geophysical Research 94 11821-11842

[13] Cappi R and Giovannozzi M 2004 Multiturn extraction and injection by means of adiabatic capture in stable islands of phase space Phys. Rev. ST-Accelerators and Beams 7024001

[14] Cary J R, Escande D F and Tennyson J L 1986 Adiabatic-invariant change due to separatrix crossing Physical Review A 34 4256-4275

[15] Cary J R and Skodje R T 1988 Reaction probability for sequential separatrix crossings Physical Review Letters 61 1795-1798 
[16] Cary J R and Skodje R T 1989 Phase change between separatrix crossings Phys. D 36 $287-316$

[17] Chirikov B V 1959 The passage of a nonlinear oscillating system through resonance Soviet Physics Doklady 4 390-394

[18] Dirac P A M 1925 The adiabatic invariance of the quantum integrals Proc. R. Soc. Lond., Ser. A, Math. Phys. Eng. Sci. 107 725-734

[19] Dolgopyat D 2012 Repulsion from resonances Mém. Soc. Math. France (N.S.) 128 p 119

[20] Freidlin M I 1998 Random and deterministic perturbations of nonlinear oscillators Doc. Math. $3223-235$

[21] Gelfreich V and Turaev D 2008 Unbounded energy growth in Hamiltonian systems with a slowly varying parameter Commun. Math. Phys. 283 769-794

[22] Goldreich P and Peale S 1966 Spin-orbit coupling in the Solar System Astron. J. 71 425-438

[23] Gurevich A V and Tsedilina E E 1985 Long Distance Propagation of HF Radio Waves (Physics and Chemistry in Space) (Berlin: Springer) p 344

[24] Kasuga T 1961 On the adiabatic theorem for the Hamiltonian system of differential equations in the classical mechanics. I, II, III Proc. Japan Acad. 37 366-371 372-376, 377-382

[25] Lifshitz I M, Slutskin A A and Nabutovskii V M 1961 The scattering of charged quasiparticles from singularities in p-space Soviet Phys. Dokl. 6 238-240

[26] Neishtadt A I 1986 Change of an adiabatic invariant at a separatrix Soviet Journal of Plasma Physics 12 568-573

[27] Neishtadt A I 1987 On the change in the adiabatic invariant on crossing a separatrix in systems with two degrees of freedom J. Appl. Math. Mech. 515 586-592

[28] Neishtadt A I 1993 On probabilistic phenomena in perturbed systems Selecta Math. Sov. 12 195-210

[29] Neishtadt A 2005 Capture into resonance and scattering on resonances in two-frequency systems Proceedings of the Steklov Institute of Mathematics 150 183-203

[30] Neishtadt A 2017 Averaging method for systems with separatrix crossing Nonlinearity 30 2871-2917

[31] Neishtadt A and Artemyev A 2012 Destruction of adiabatic invariance for billiards in a strong nonuniform magnetic field Physics Review Letters 108064102

[32] Neishtadt A, Sidorenko V and Treschev D 1997 Stable periodic motions in the problem on passage through a separatrix Chaos 7 2-11 
[33] Neishtadt A, Simo C, Treschev D and Vasiliev A 2008 Periodic orbits and stability islands in chaotic seas created by separatrix crossings in slow-fast systems Discrete and Continuous Dynamical Systems - Series B 10 621-650

[34] Neishtadt A, Vainchtein D and Vasiliev A 2008 Adiabatic invariance in volume-preserving systems In: IUTAM Symposium on Hamiltonian Dynamics, Vortex Structures, Turbulence IUTAM Bookseries, vol 6 (Dordrecht: Springer) 89-107

[35] Neishtadt A and Vasiliev A 2005 Phase change between separatrix crossings in slow-fast Hamiltonian systems Nonlinearity 18 1393-1406

[36] Northrop T G 1963 The Adiabatic Motion of Charged Particles New York: Interscience Publishers p 109

[37] Robnik M and Berry M V 1985 Classical billiards in magnetic fields Journal of Physics A 18 1361-1378

[38] Shah K, Turaev D, Gelfreich V and Rom-Kedar V 2017 Equilibration of energy in slow - fast systems Proc. Natl. Acad. Sci. USA 114 E10514

[39] Timofeev A V 1978 On the constancy of an adiabatic invariant when the nature of the motion changes Sov. Phys., JETP 48 656-659

[40] Vasiliev A, Neishtadt A and Artemyev A 2011 Nonlinear dynamics of charged particles in an oblique electromagnetic wave Physics Letters A 375 3075-3079

[41] Wisdom J 1985 A perturbative treatment of motion near the $3 / 1$ commensurability Icarus $63272-289$

[42] Wolansky G 1990 Limit theorem for a dynamical system in the presence of resonances and homoclinic orbits J. of Differ. Equat. 83, 2, 300-335

[43] Zelenyi L M, Neishtadt A I, Artemyev A V, Vainchtein D L and Malova H V 2013 Quasiadiabatic dynamics of charged particles in a space plasma Physics-Uspekhi 56 347-394 